\title{
Variable-Rate Pumping Tests for Radially Symmetric Nonuniform Aquifers
}

\author{
JAMes J. Butler, Jr., and CARL D. MCElwee \\ Kansas Geological Survey, The University of Kansas, Lawrence
}

\begin{abstract}
Conventional pumping test methodology is of limited effectiveness for defining the spatial distribution of aquifer properties because of the nonuniqueness of the parameter estimates. Sensitivity analysis can be used to develop a pumping test procedure that significantly decreases the uncertainty associated with the estimated parameters. This approach employs systematic variations in pumpage rates to achieve reductions in parameter uncertainty. These reductions are obtained by increasing the sensitivity of drawdown to flow properties while simultaneously constraining the growth in the correlation between the effects of different flow properties on observation well drawdown. Numerical experiments demonstrate the importance of the magnitude and frequency of the rate variations, the spatial and temporal pattern of data collection, as well as the dependence of the technique on the total duration of the pumping test. Significant decreases in parameter uncertainty can be expected in any flow system in which the primary component of flow is in the radial direction. This study demonstrates that sensitivity analysis can be an important tool in the development of methodology for the characterization of subsurface properties.
\end{abstract}

\section{INTRODUCTION}

Analyses of pumping test drawdowns have traditionally been used to estimate volumetric averages of the storage and transmissive properties of a subsurface unit. Neither the form of this average nor the volume over which it is taken is explicitly defined. In addition, most analyses assume that aquifer properties are uniform in space, resulting in an unknown amount of error being introduced into parameter estimates for the nonuniform aquifers of natural systems. Recently, the usefulness of the single-valued parameters resulting from a conventional pumping test analysis has been questioned [e.g., de Marsily, 1987]. This questioning is in keeping with the increased awareness that has arisen in the last decade of the importance of spatial variations in subsurface properties. Clearly, an analysis that would yield an estimate of the spatial distribution of flow properties would be preferred. This article describes an approach for the performance and analysis of pumping tests that provides an estimate of the large-scale property variations in a unit. The advantages of this approach over current techniques are demonstrated for the ideal case of radially symmetric nonuniform aquifers.

Drawdown at an observation well in a radial flow field at different times during a pumping test reflects conditions in different portions of the aquifer. It can be shown that the flow properties of a specific portion of an aquifer only significantly influence changes in drawdown for a time of quite limited duration. Butler [1986, 1988] discusses these ideas and their ramifications for pumping test analysis. Sensitivity analysis is employed here to explore in more detail the nature of the relationship between drawdown at an observation well and flow properties in different portions of an aquifer.

A pumping test approach for estimation of the spatial distribution of flow properties must be designed to take advantage of the transient dependence of changes in drawdown on the flow properties in different portions of an aquifer. The methodology described here employs con-

Copyright 1990 by the American Geophysical Union.

Paper number 89WR01580.

0043-1397/90/89WR-01580\$05.00 trolled variations in pumping rates to obtain multiple samples of flow behavior in each portion of an aquifer. These multiple samples allow the effects of flow properties in a specific portion of the aquifer to be more readily distinguished from the effects of properties in other zones and from the background noise due to measurement error. A pattern of rate variations can be defined that allows the distribution of flow properties both in the vicinity of and at a distance from the pumping well to be estimated to a greater accuracy than normally obtainable. The spatial and temporal strategy for collection of drawdown data is shown to be a critical factor in the success of the proposed methodology.

There has been relatively little work to date on the development of analytical solutions for use in pumping test analysis in nonuniform units. Streltsova [1988] provides a review of the major contributions in this area. The groundwater literature is replete, however, with descriptions of applications of numerical flow models to pumping test analysis in nonuniform systems. These numerical models, in general, provide for greater flexibility than their analytic counterparts. Rushton and Chan [1977] were among the early workers in this area, presenting a numerical model specifically designed for use in pumping test analysis. Their approach employs manual adjustment of parameters to obtain a satisfactory agreement between simulated and measured drawdowns. McElwee [1982a] has proposed a more flexible model, using sensitivity analysis [McElwee, 1987] to perform the parameter adjustments automatically. Regardless of whether a model specifically designed for pumping test analysis or a more general code is employed, these numerical approaches have been found to be of limited effectiveness because of the nonuniqueness of model parameters. Nonuniqueness allows the same drawdown behavior to be duplicated by a large number of possible parameter vectors, each with a similar likelihood of representing the actual system. Yeh and Sun [1984] are among the few to have addressed this problem of nonuniqueness in a pumping test context, finding the group of acceptable parameter vectors given a future use for the results. The approach described here attempts to significantly narrow the group of acceptable parameter vectors resulting from a pumping test analysis through controlled variations in the pumping rate. 
The parameter nonuniqueness that hinders pumping test analyses is a result of a number of factors including observation well drawdown data being insensitive to flow properties in portions of the model domain, a high degree of correlation between the effects of different flow properties on observation well drawdown, and measurement error. The effects of drawdown insensitivity to flow properties and the impact of correlation are the primary focus of this discussion. McElwee [1982 b], among others, has discussed the effects of measurement error. This article outlines a pumping test approach based on the design of a more appropriate data collection strategy and the manipulation of the schedule of pumping in order to decrease the impact of parameter nonuniqueness on pumping test analysis. Although the approach is demonstrated for the ideal case of a radially symmetric nonuniform aquifer, these concepts should have considerable relevance for more complex systems.

\section{Drawdown and Its Sensitivity to Flow Properties}

In order to define appropriate pumping test strategies, the nature of drawdown at an observation well and its relation to the storage and transmissive properties of an aquifer must be considered in some detail. McElwee and Yukler [1978] have previously assessed the relationship between drawdown and flow properties in the uniform-aquifer case. Based on simulated response data and a simple analytical solution, Butler $[1986,1988]$ has proposed an interpretation of drawdown at an observation well in a radial-flow field that considers the effect of spatial variations in flow properties. That interpretation is extended here using the more rigorous framework of sensitivity analysis. This investigation begins by considering behavior in near-uniform systems, followed by an analysis of behavior in more strongly nonuniform systems. In order to elucidate general principles, the discussion is limited to flow in a single radial dimension.

The confined, one-dimensional flow of groundwater to a pumping well can be represented by the following equation:

$$
\frac{\partial}{\partial r}\left(T(r) \frac{\partial s}{\partial r}\right)+\frac{T(r)}{r} \frac{\partial s}{\partial r}=S(r) \frac{\partial s}{\partial t}
$$

with

$$
\begin{gathered}
s(r, 0)=0 \quad r_{w} \leq r<\infty \\
s(\infty, t)=0 \quad t>0 \\
2 \pi r_{w} T\left(\frac{\partial s}{\partial r}\right)_{r_{w}}=-Q \quad t>0
\end{gathered}
$$

where

$T(r)$ transmissivity, $\left[L^{2} / T\right]$;

$S(r)$ storage coefficient (storativity), dimensionless;

$s(r, t)$ drawdown, $[L]$;

$r$ radial direction, $[L]$;

$r_{w}$ radius of pumping well, $[L]$;

$Q$ pumpage from well, $\left[L^{3} / T\right]$;

$t$ time, [T].

Solution of (1) yields the drawdown, as a function of radial distance and time, in response to defined initial and boundary conditions (2), and given distributions of aquifer properties. The sensitivity of drawdown at a given observation

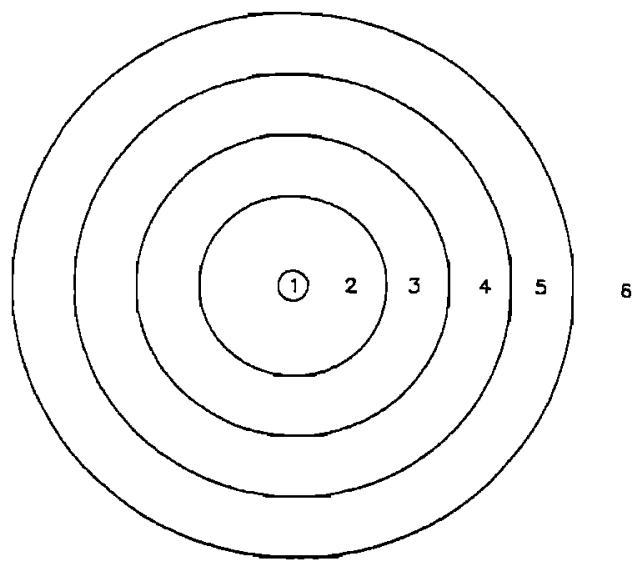

Fig. 1. Hypothetical aquifer employed in the simulations of this work. Pumping well is located at the center of zone 1; remaining zones are concentric rings about this well (not to scale).

point to transmissivity and storage at any point within the aquifer can be calculated using the following equations [McElwee, 1987]:

$$
\begin{aligned}
& \frac{\partial}{\partial r}\left(T(r) \frac{\partial U_{T}\left(r, t ; r_{s}\right)}{\partial r}\right)+\frac{T(r)}{r} \frac{\partial U_{T}\left(r, t ; r_{s}\right)}{\partial r} \\
& +\frac{1}{r} \frac{\partial}{\partial r}\left(r \Delta\left(r-r_{s}\right) \frac{\partial s}{\partial r}\right)=S(r) \frac{\partial U_{T}\left(r, t ; r_{s}\right)}{\partial t}
\end{aligned}
$$

$$
\begin{aligned}
\frac{\partial}{\partial r}\left(T(r) \frac{\partial U_{S}\left(r, t ; r_{s}\right)}{\partial r}\right) & +\frac{T(r)}{r} \frac{\partial U_{S}\left(r, t ; r_{s}\right)}{\partial r} \\
& =S(r) \frac{\partial U_{S}\left(r, t ; r_{s}\right)}{\partial t}+\Delta\left(r-r_{s}\right) \frac{\partial s}{\partial t}
\end{aligned}
$$

where

$$
\begin{aligned}
U_{T}\left(r, t ; r_{s}\right) \quad \begin{array}{l}
\text { sensitivity of drawdown at }(r, t) \text { to } \\
\text { transmissivity at } r_{s}, \text { equal to } \partial s(r, t) / \partial T\left(r_{s}\right), \\
{[T / L] ;}
\end{array} \\
U_{s}\left(r, t ; r_{s}\right) \begin{array}{l}
\text { sensitivity of drawdown at }(r, t) \text { to storativity } \\
\text { at } r_{s}, \text { equal to } \partial s(r, t) / \partial S\left(r_{s}\right),[L] ;
\end{array} \\
\Delta\left(r-r_{s}\right) \begin{array}{l}
\text { a modified dimensionless delta function, } \\
\text { equal to } 1 \text { when } r=r_{s}, \text { zero otherwise. }
\end{array}
\end{aligned}
$$

The similarity of the form of equations (3) and (4) to (1) enabled the same computer code used for solution of (1) to be utilized for sensitivity analysis following minimal modifcation. In this paper, $U_{T}$ and $U_{S}$ are referred to as either sensitivity coefficients or simply the sensitivity.

The relationship between drawdown and flow properties is examined here in the radial configuration displayed in Figure 1. In this case, a pumping well is assumed sited at the center of a patch of material (zone 2) of higher permeability than that in the surrounding region. In the immediate vicinity of the well, a zone of lower permeability (zone 1) is assumed to reflect the influence of a less transmissive well skin. Zones 3-5 are assumed to provide a transition between the more permeable patch and regions where permeability values are more representative of the aquifer as a whole. Although clearly a simplification, this configuration is considered a reasonable representation of conditions that might be met in the field. The variation between flow properties in adjacent 
TABLE 1. Radial Boundaries and Transmissivities for the Zones of the Hypothetical Aquifer of Figure 1

\begin{tabular}{ccccc}
\hline & & & \multicolumn{2}{c}{$T, \mathrm{~m}^{2} / \mathrm{d}$} \\
\cline { 4 - 5 } Zone & $r_{1}, \mathrm{~m}$ & $r_{2}, \mathrm{~m}$ & Case 1 & Case 2 \\
\hline 1 & 0.05 & 0.638 & 1564. & 86.4 \\
2 & 0.638 & 29.04 & 1728. & 1728. \\
3 & 29.04 & 75.43 & 1642. & 864. \\
4 & 75.43 & 142.5 & 1572. & 172.8 \\
5 & 142.5 & 269.4 & 1564. & 86.4 \\
6 & 269.4 & 43810. & 1557. & 17.28 \\
\hline
\end{tabular}

Storativity is set to a constant value of 0.0005 for both cases; $r_{1}$, radius of inner zonal boundary, and $r_{2}$, radius of outer zonal boundary.

zones, summarized as case 1 in Table 1 , is initially kept small in order to examine behavior in a system where the effects of aquifer zonation can be clearly illustrated.

Figure 2 is a plot of the sensitivity of drawdown near the pumping well (within zone 1) to transmissivity in zones 2,4 , and 6 under conditions of constant pumpage $\left(1000 \mathrm{~m}^{3} / \mathrm{d}\right)$. Note that there is a finite interval of time during which changes in drawdown are sensitive to conditions in zones 2 and 4. After that interval has passed, further changes in drawdown are independent of transmissivity in those zones, as reflected by the near-constant sensitivity coefficients at large times. This finding regarding the independence of the change in drawdown at large times from near-well properties is in agreement with existing analytical results [e.g., Barker and Herbert, 1982; Butler, 1988]. Essentially, as long as the front of the cone of depression is within a given zone (i.e., the head gradient across the zone has not reached a nearconstant value), the sensitivity of drawdown to transmissivity in that zone will continue to increase. The flattening of the sensitivity curve indicates that further changes in drawdown contribute little information about the transmissivity in that zone (i.e., the head gradient across the zone has reached a near-constant value), with the level at which the curve flattens being an indication of the relative size of the zone with respect to its position in the radial-flow field (cf. T2 and

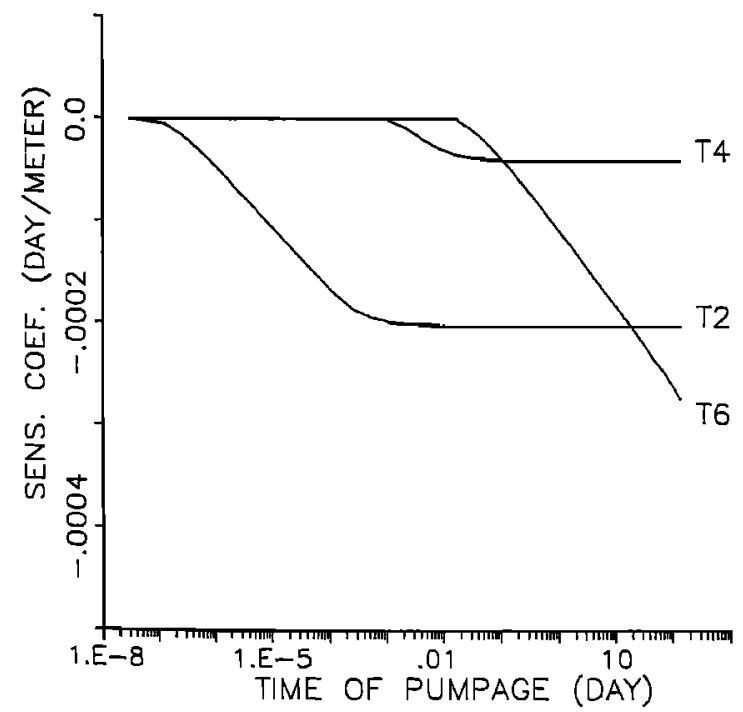

Fig. 2. Sensitivity versus time plot for drawdown in zone 1. Sensitivities to the transmissivities of zones 2,4 , and 6 are plotted.

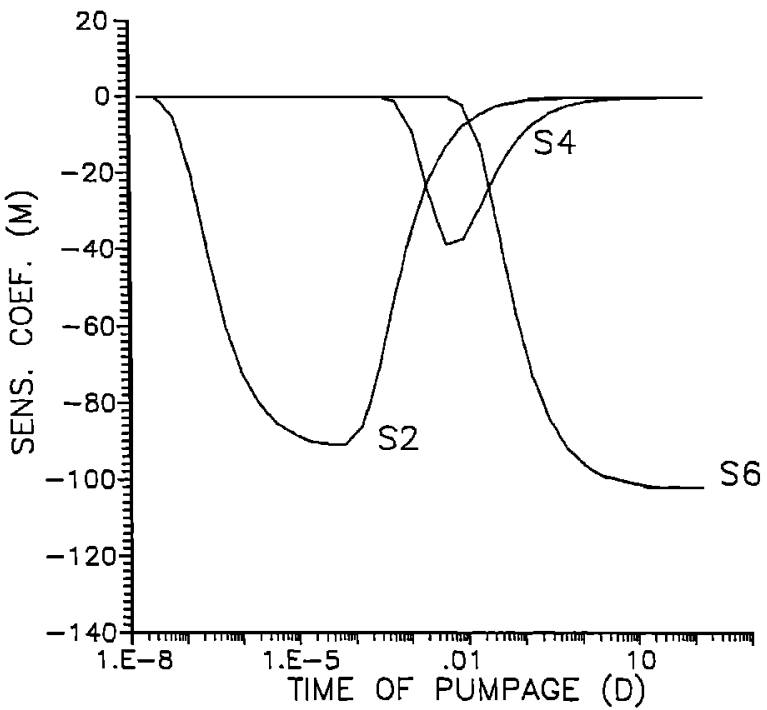

Fig. 3. Sensitivity versus time plot for drawdown in zone 1 . Sensitivities to the storativities of zones 2,4 , and 6 are plotted.

T4 curves of Figure 2). Note that the sensitivity of drawdown to transmissivity in zone 6 is a product of the essentially infinite-aquifer conditions assumed here and is equivalent to the results of McElwee and Yukler [1978], which show that the sensitivity of drawdown to transmissivity continually increases in a uniform system.

The sensitivity of drawdown to storativity displays a markedly different behavior. As shown in Figure 3, the sensitivity of drawdown to storativity in a given finite zone goes to zero at large times, indicating that drawdown at large times, and not just its changes, is independent of the storativity of material near the pumping well. In this case, the sensitivity plot is a function of behavior during the period of transition when the front of the cone of depression is moving from one zone to another. As the front of the cone of depression moves into a zone, the curve portraying the sensitivity of drawdown to storage in that zone will display very large increases in magnitude with time. In zones of large size, relative to their radial position, the curve characterizing the sensitivity of drawdown to storativity will reach a maximum magnitude that is maintained until the effects of the outer zonal boundary are felt. As the cone front moves out of the zone, the sensitivity curve will display a rapid decrease in magnitude to zero. In an infinite uniform aquifer, the sensitivity curve maintains its maximum magnitude for the duration of pumpage [McElwee and Yukler, 1978], as demonstrated by the $S 6$ curve of Figure 3.

The preceding discussion has focused on the effects of aquifer zonation on sensitivity relationships. The sensitivity of drawdown to flow properties, however, is also a function of the magnitude of both the flow properties and their interzonal variations. McElwee and Yukler [1978] derived the following analytical expressions for the sensitivity coefficients in the case of a uniform aquifer:

$$
U_{T}=-\frac{s}{T}+\frac{Q}{4 \pi T^{2}} \exp \left(-\frac{r^{2} S}{4 T t}\right)
$$




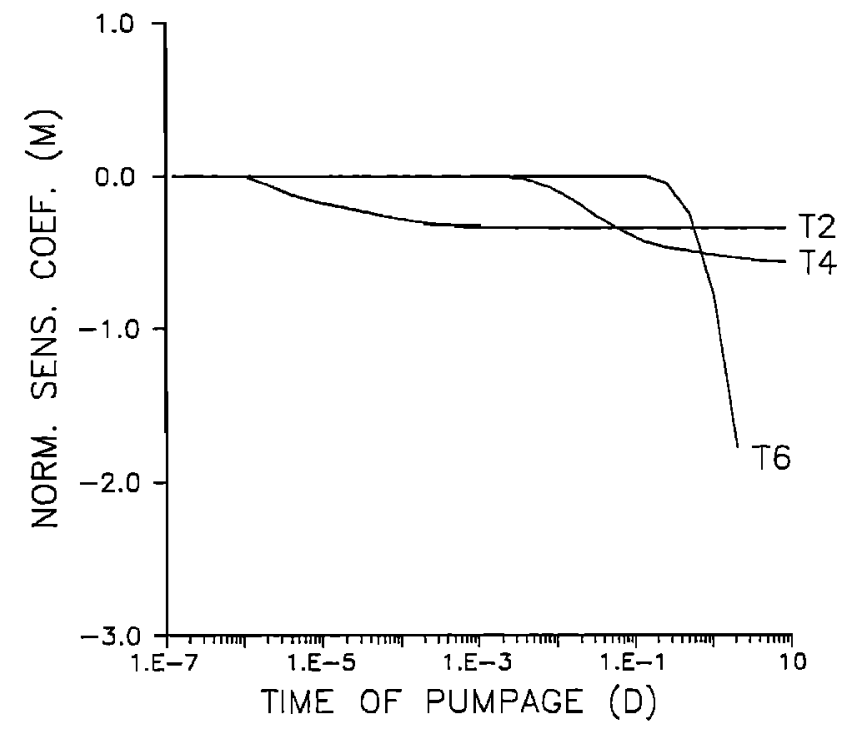

Fig. $4 a$

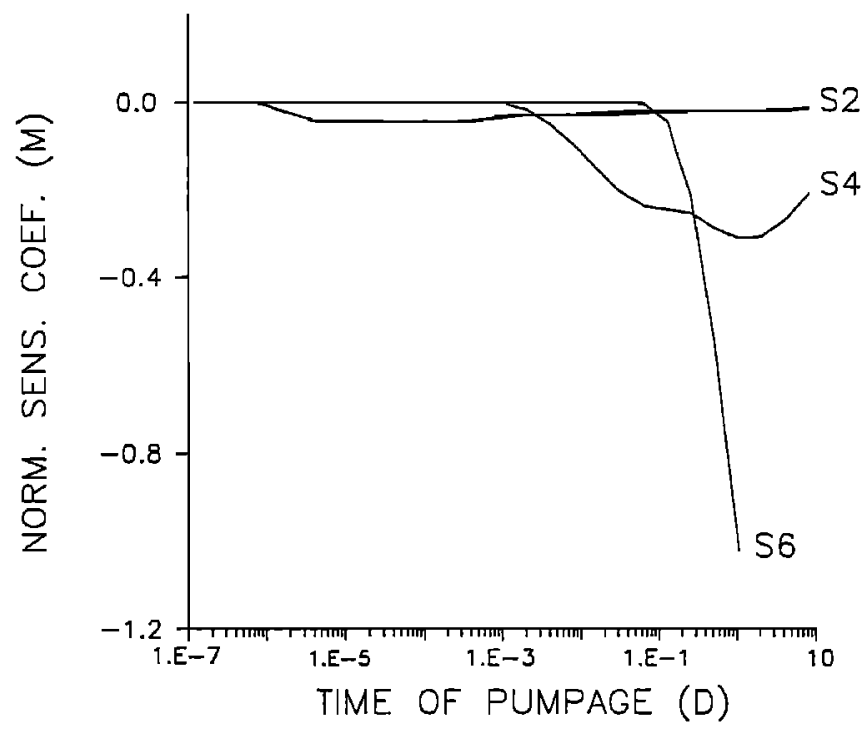

Fig. $4 b$

Fig. 4. Sensitivity versus time plots for configuration of case 2 (sensitivities normalized by multiplication by respective model parameters), drawdown measured in zone 1. (a) Plot of drawdown sensitivity to the transmissivities of zones 2,4 , and 6. (b) Plot of drawdown sensitivity to the storativities of zones 2,4 , and 6 .

$$
U_{S}=\frac{-Q}{4 \pi T S} \exp \left(-\frac{r^{2} S}{4 T t}\right)
$$

where

$$
s=\frac{Q}{4 \pi T} \int_{\left(r^{2} S / 4 T t\right)}^{\infty} \frac{e^{-u}}{u} d u
$$

The sensitivity of drawdown to storativity is inversely dependent on both transmissivity and storativity, whereas the sensitivity of drawdown to transmissivity quickly becomes virtually independent of storativity. Relationships analogous to (5) and (6) can be shown to be characteristic of radially symmetric nonuniform systems as well. Sensitivity coeffi-

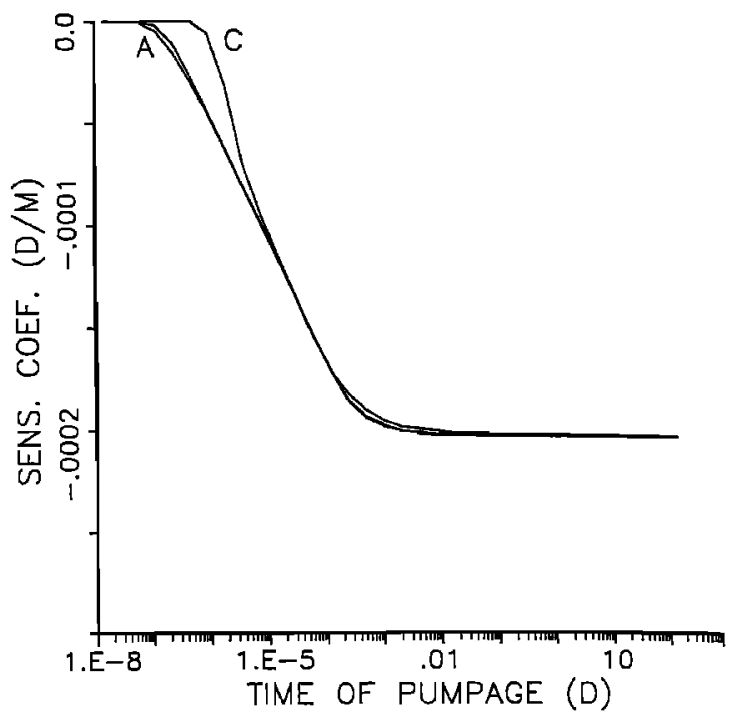

Fig. $5 a$

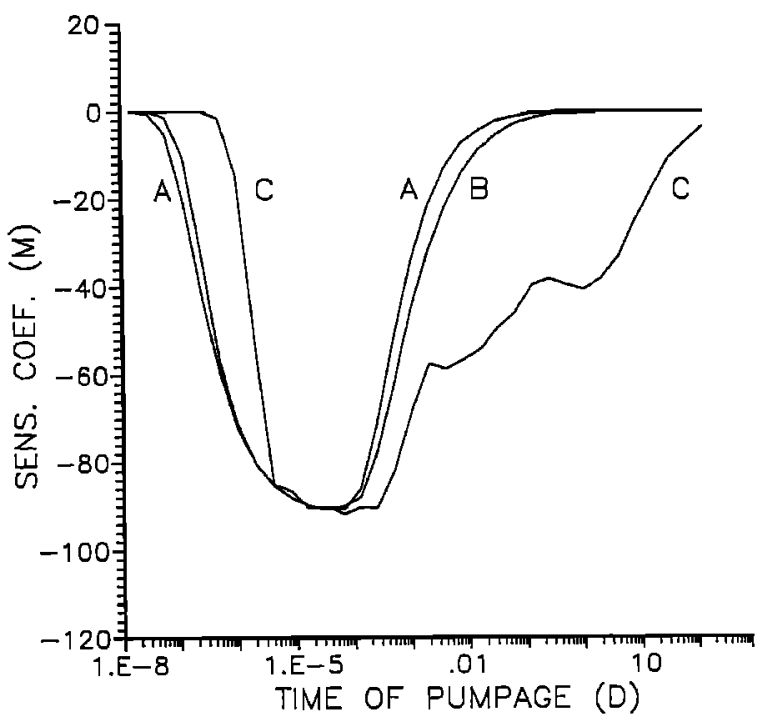

Fig. $5 b$

Fig. 5. Sensitivity versus time plots showing the dependence on interzonal variations in transmissivity (transmissivity patterns explained in text), drawdown measured in zone 1. (a) Plot of the sensitivity of drawdown to zone 2 transmissivity. (b) Plot of the sensitivity of drawdown to zone 2 storativity.

cients can be normalized by multiplying through by the respective flow properties. The normalized coefficients can then be used to compare sensitivities to different parameters in nonuniform systems. As shown by (5) and (6), systems with lower transmissivities will have higher normalized sensitivities and thus will, in general, be more amenable to inversing analyses, given that a sufficient time of pumpage has elapsed [McElwee, 1987]. Figure 4 displays the normalized sensitivity plots for the configuration of case 2 of Table 1 under constant pumpage conditions $\left(1000 \mathrm{~m}^{3} / \mathrm{d}\right)$. Note how the decrease in the transmissivity of the outer zones alters the relationships of Figures 2 and 3.

Interzonal variations in flow properties have a considerable impact on sensitivity relationships, especially with 


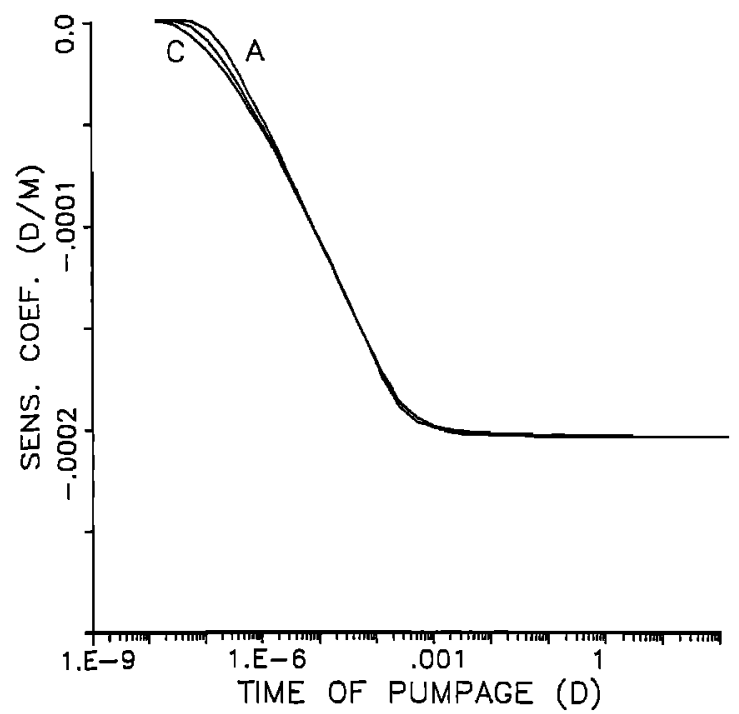

Fig. $6 a$

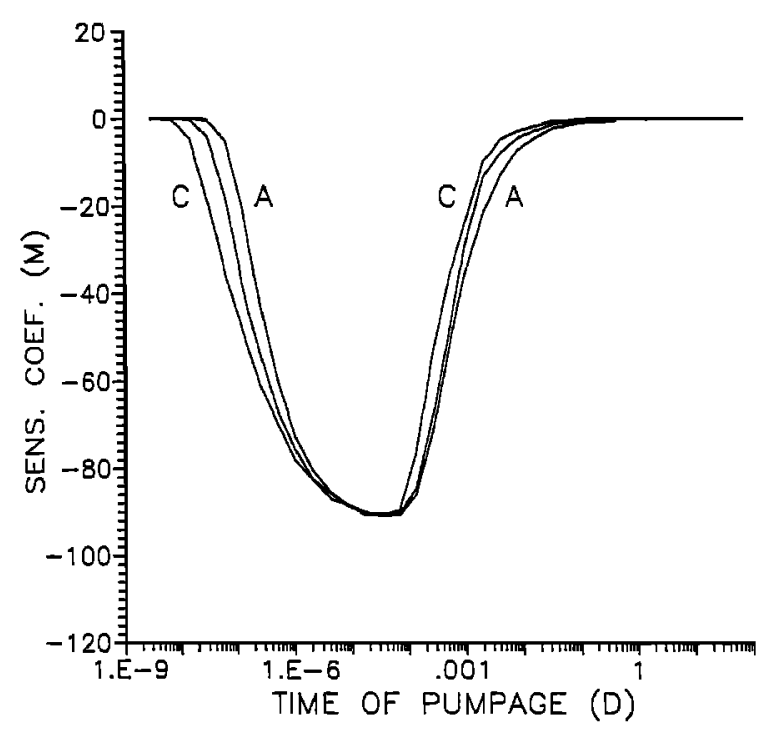

Fig. $6 b$

Fig. 6. Sensitivity versus time plots showing the dependence on interzonal variations in storativity (storativity patterns defined in Table 2), drawdown measured in zone 1. (a) Plot of the sensitivity of drawdown to zone 2 transmissivity. (b) Plot of the sensitivity of drawdown to zone 2 storativity.

respect to the sensitivity of drawdown to storativity. Figure 5 is a pair of plots depicting the sensitivity of drawdown near the pumping well to flow properties in zone 2 as a function of the interzonal variations in transmissivity. Three different magnitudes of transmissivity variations are examined: the variations of case 1 (curve $A$ ), in which the variations between zone 2 and the other zones are approximately $10 \%$ of those of case 2; a configuration in which the variations in transmissivity between zone 2 and the other zones are approximately $50 \%$ of those of case 2 (curve B); and the variations of case 2 (curve $C$ ). The transmissivity variations of case 2 are in keeping with the magnitudes reported by Barker and Herbert [1982] and Toth [1973] and are considered to be representative of variations more extreme than those observed in most aquifers. Storativity is kept constant at 0.0005 for all zones in these three cases. Note that the sensitivity of drawdown to zone 2 transmissivity is relatively unaffected by the transmissivity of other zones except that of zone 1 , which determines the time at which the properties of zone 2 initially affect drawdown. This insensitivity is due to the fact that once the head gradient across a zone has been established, it is essentially unaffected by the properties of more distant zones. As shown by Figure $5 b$, however, the sensitivity of drawdown to the storativity of zone 2 may be very strongly affected by decreases in transmissivity in the zones beyond zone 2 . This is a reflection of behavior during the periods of transition when the front of the cone of depression is moving across zonal boundaries. The drawdown behavior during these transition periods is controlled by the differences in flow properties of material on either side of a zonal boundary. In curve $C$ of Figure $5 b$, transmissivity decreases in zone 3 without a proportional decrease in storativity. The resulting decrease in diffusivity acts to slow the propagation of the cone front and to lengthen the period of transition between zones 2 and 3. The longer transition period, in conjunction with the lower transmissivities of the outer zones, results in the transitions into zones 4 and 5 affecting drawdown behavior before the cone front has completely moved out of zone 2 . These outer zonal transitions produce the two kinks in the ascending portion of curve C. Large increases in transmissivity beyond zone 2 produce a significantly different result. In that case, the curve depicting the sensitivity of drawdown to zone 2 storativity rapidly goes to zero due to the inverse relationship between transmissivity and drawdown sensitivity (see equation (6)).

Figure 6 is a pair of plots depicting the sensitivity of drawdown to zone 2 properties as a function of interzonal variations in storativity. Three different magnitudes of storativity variations are examined as shown in Table 2 ; in each case the zonal transmissivities are those of case 1. Relatively little information exists concerning variations in the storativity of natural systems, so the variations of Table 2 attempt to bound those that might be observed in confined aquifers in the field. The plots of Figure 6 demonstrate that large increases in storativity outward from zone 2 have a rather minimal impact on the sensitivity of drawdown in zone 1 to flow properties in zone 2 . Once again, the propagation of the cone front is slowed owing to decreases in diffusivity in zones outward from zone 2 . However, despite this decrease in diffusivity, the inverse relationship between storativity and drawdown sensitivity (see equation (6)) results in sensitivities to storativity going to zero more rapidly than in the constant-storativity case.

TABLE 2. Three Different Patterns of Interzonal Variations in Storativity for the Hypothetical Aquifer of Figure 1

\begin{tabular}{llll}
\hline Zone* & $S_{a}$ & \multicolumn{1}{c}{$S_{b}$} & \multicolumn{1}{c}{$S_{c}$} \\
\hline 1 & 0.0005 & 0.00025 & 0.0001 \\
2 & 0.0005 & 0.0005 & 0.0005 \\
3 & 0.0005 & 0.00056 & 0.001 \\
4 & 0.0005 & 0.00158 & 0.005 \\
5 & 0.0005 & 0.00178 & 0.01 \\
6 & 0.0005 & 0.005 & 0.05 \\
\hline
\end{tabular}

* Zonal boundaries and transmissivities (case 1) as in Table 1. 


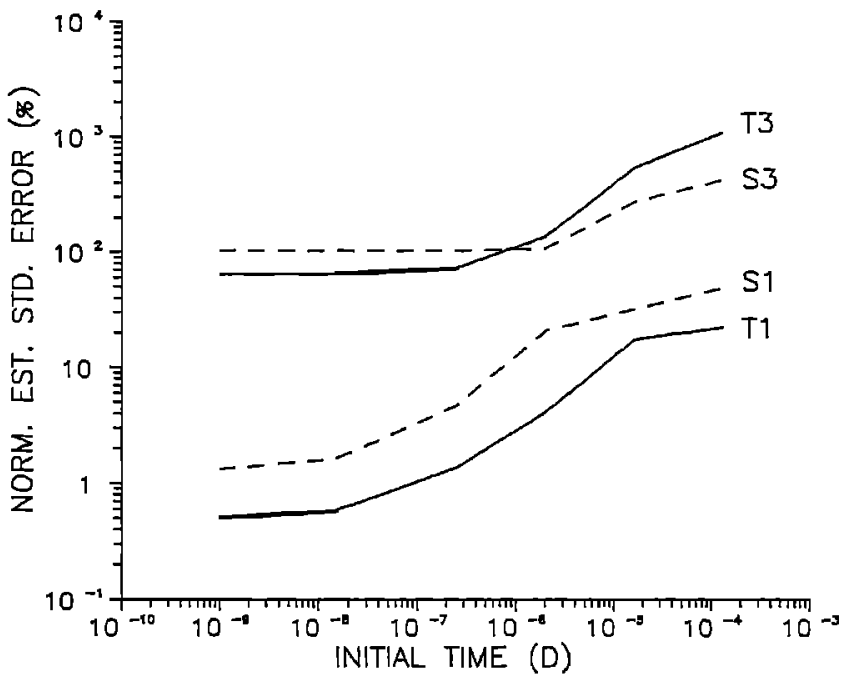

Fig. 7. Plot of estimated standard error for zone 1 and 3 parameters versus initial time of data collection for drawdown measured in zone 1 .

\section{Estimation of Aquifer Properties From Measured DRAWDOWN}

In order to explore the impact of sensitivity relationships in nonuniform systems on inverse analyses, a series of pumping test simulations was performed using the idealized configuration of case 2 . Parameters were estimated from the simulated drawdowns uising an unweighted least squares routine that employs sensitivity coefficients (see Appendix A). The estimated standard error [Beck and Arnold, 1977] arising from the least squares analysis was employed as a measure of the uncertainty in the parameter estimates. The estimated standard errors of this work were normalized by dividing by the corresponding parameter estimate and are expressed as a percent. Note that for all cases, the simulated drawdowns were rounded to the nearest centimeter before application of the least squares routine.

The first set of simulations was designed to assess the dependence of the estimated standard error on the initial time and the frequency of data collection, two aspects of a pumping test that Figures $2-4$ imply could be of significant importance in nonuniform systems. Drawdown measurements were assumed taken at the pumping well at every time step of the simulations, with the interval between time steps increasing by a factor of 2 between successive steps (time step acceleration factor of 2.0). Figure 7 illustrates the effect of the initial time of data collection on parameter estimates for a pumping test of approximately 1 day in duration. Significant increases in the estimated standard error of parameters of zones near the pumping well are seen with increases in the initial time of data collection. Clearly, collection of drawdown data must commence at a very early time if spatial variations in flow properties relatively close to the pumping well are to be defined. For the remaining simulations of this work, an initial time of approximately one tenth of a second $\left(1.0 \times 10^{-6}\right.$ day $)$ was employed.

In Figure 8 the effect of the frequency of data collection is illustrated by plotting the estimated standard error versus the time step acceleration factor employed in the pumping test simulation. In each of the simulations of Figure 8, the total time of the pumping test was approximately 1 day. The

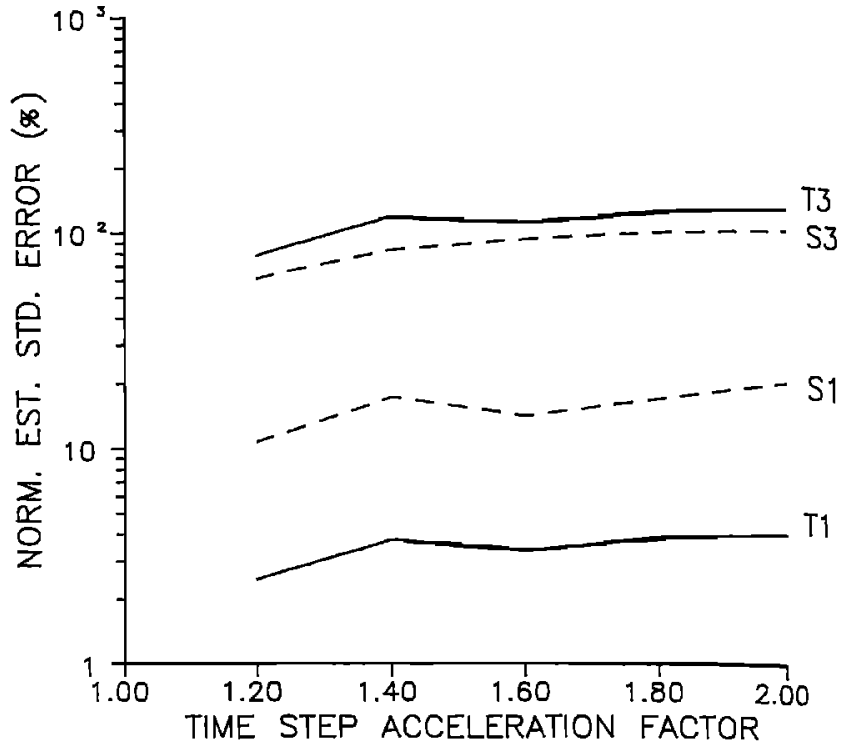

Fig. 8. Plot of estimated standard error for zone 1 and 3 parameters versus temporal frequency of measurement for drawdown in zone 1 (a time step acceleration factor of 1.2 corresponds to 12-13 measurements per log cycle; a factor of 2.0 corresponds to $3-4$ measurements per $\log$ cycle); initial time of data collection is approximately $1 \times 10^{-6}$ day.

initial time varied a small amount between simulations in order to ensure a near-constant total time. These differences in initial time were largely responsible for the kinks in the curves of Figure 8. Comparison of Figures 7 and 8 shows that the standard error is much less dependent on the frequency of data collection than on the initial time, for the data collection frequencies of potential use in a conventional pumping test. Our interpretation of these results is that for zones close to the pumping well, large initial times of data collection result in the effects of the transmissivity in several adjacent zones becoming inseparable and the effects of the storativity in certain zones being ignored. In the case of sampling rates, the time interval between drawdown measurements must be very large before similar behavior is observed.

Even in the presence of a small initial time and a reasonable sampling rate, pumping test analyses in nonuniform aquifers are still of quite limited effectiveness owing, in large part, to measurement error, the absolute magnitude of the sensitivity coefficients, and the correlation between the effects of different flow properties on observation well drawdown. The relative magnitude of the sensitivity coefficients compared to the measurement error is clearly a critical consideration. If the absolute values of the sensitivity coefficients are small with respect to the measurement error, it is difficult to dampen the effect of measurement error and obtain a reliable parameter estimate. In the case of zonal transmissivity, it can be shown (see Appendix B) that once the cone front has passed out of the zone of interest, the estimated standard error of the zonal transmissivity can decrease no faster than a rate approximately inversely proportional to the square root of the total number of drawdown measurements from an observation well. In addition, this decrease may be considerably slowed by an increase in sensitivity correlation (see Appendix A), which 
often accompanies such situations. Thus it may not be feasible to collect the number of measurements required to obtain a significantly better estimate of zonal transmissivity once the cone front has passed out of the zone of interest. In the case of zonal storativity, the effect of measurement error is even more difficult to minimize because of the finite duration of time during which drawdown is sensitive to the storativity of a particular zone. Clearly, therefore, an approach that considerably increases the sensitivity of drawdown to flow properties in different portions of the aquifer without producing a significant increase in the degree of correlation between sensitivity coefficients could be of considerable use. The following section outlines such an approach.

\section{Variable-Rate Pumping Tests}

One simple way to increase the sensitivity of drawdown to zonal properties is to pursue a pumping strategy consisting of a series of rate increases. Each time the pumping rate is increased, a new cone of depression, superimposed on the original one, propagates out from the pumping well, producing an increase in sensitivity and a new interval of time during which a given zone influences changes in drawdown. A pattern of continual increases in pumpage, however, produces a significant increase in the correlation between the responses of drawdown to different zonal properties, as is shown later. A variable-rate pumping procedure is introduced here that allows significant increases in sensitivity without incurring correspondingly large increases in correlation. In order to understand this approach, the effect of pumping rate changes on sensitivity and correlation relationships must be examined. Note that the correlation between the effects of different flow properties on observation well drawdown is quantified here using the "pseudo" correlation between the diagonal elements of the sensitivity summation matrix of (A3). Although the correlation between sensitivity coefficients is, as shown in Appendix A, the basis of parameter correlation, the relationship between sensitivity and parameter correlation is complicated. The correlation involving sensitivity coefficients is considerably easier to interpret and thus is used in this article.

Figure 9 illustrates the effect of a series of pumping rate increases on the sensitivity of drawdown near the pumping well to flow properties in zones 2, 4, and 6 of case 2 (see Figure 4 for the constant-pumpage ( $Q=1000 \mathrm{~m}^{3} / \mathrm{d}$ ) case). Each rate increase generally produces a considerable increase in the absolute value of the sensitivity coefficient and a finite period of time during which changes in drawdown are heavily influenced by properties in a particular zone. Thus it would seem that by continually increasing the rate of pumpage, the sensitivity of drawdown to zonal properties could be increased significantly, leading to a sizable decrease in the estimated standard error of zonal parameters. Note that the sharpness of the response of sensitivity coefficients to a rate change is dampened as zones at progressively greater distances from the pumping well are considered. In the case of zone 6 , its size and distance from the pumping well results in a sensitivity plot in which the effects of individual rate changes are difficult to discern.

The pattern of pumping rate variations depicted in Figure $9 c$ cannot be pursued for an extended period of time, since pumpage obviously cannot be continually increased. In

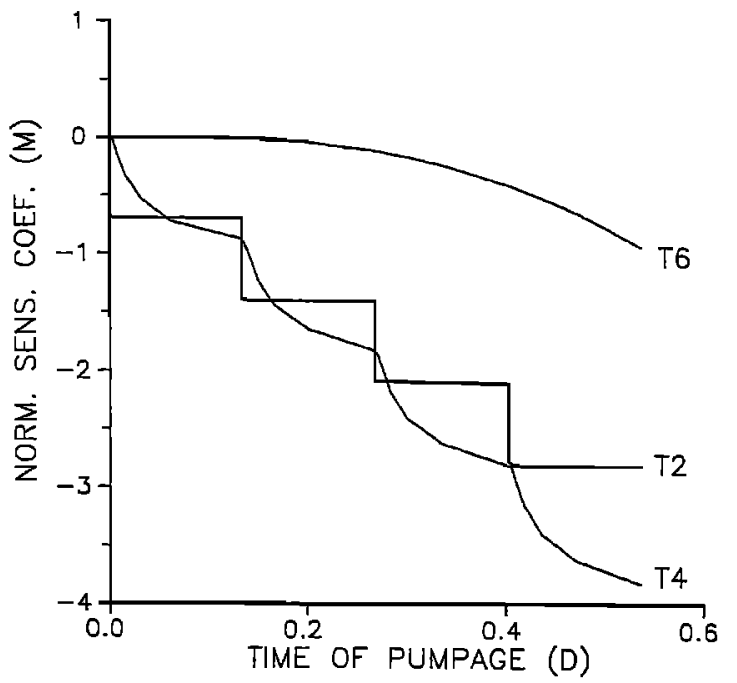

Fig. $9 a$

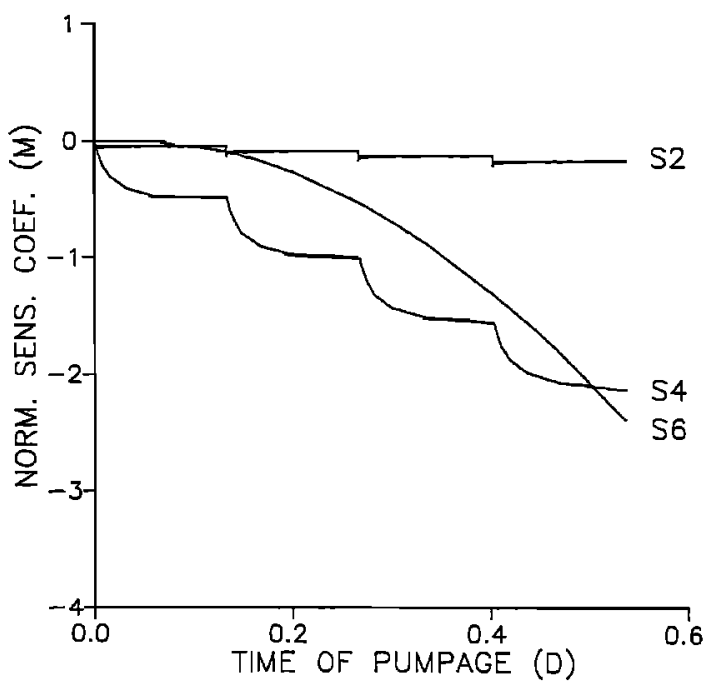

Fig. $9 b$

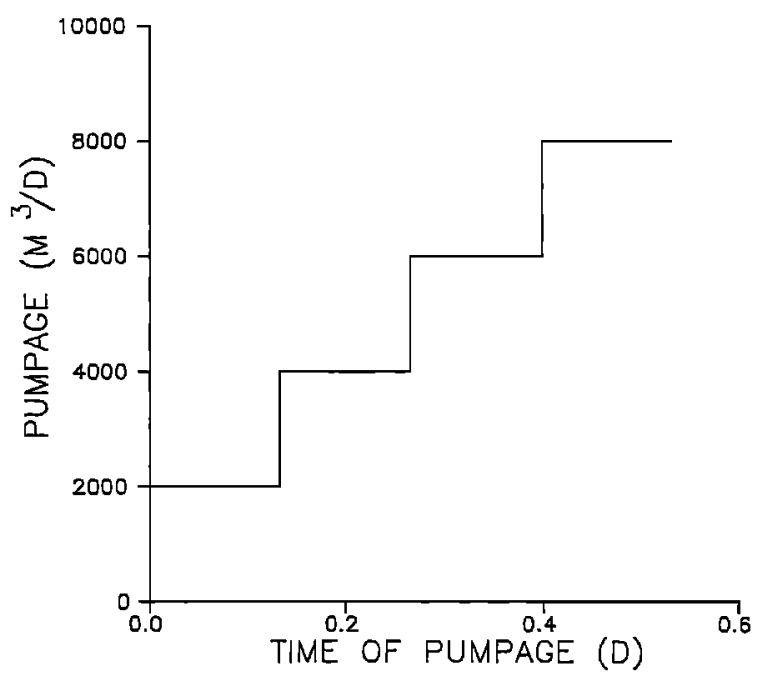

Fig. $9 c$

Fig. 9. (a) Plot of sensitivity versus time for transmissivities in zones 2,4 , and 6 , drawdown measured in zone 1 (sensitivities normalized by multiplication by respective model parameters). (b) Plot of sensitivity versus time for storativities in zones 2,4 , and 6 , drawdown measured in zone 1. (c) Pumpage versus time plot for the case of increasing pumpage every 3.2 hours. 


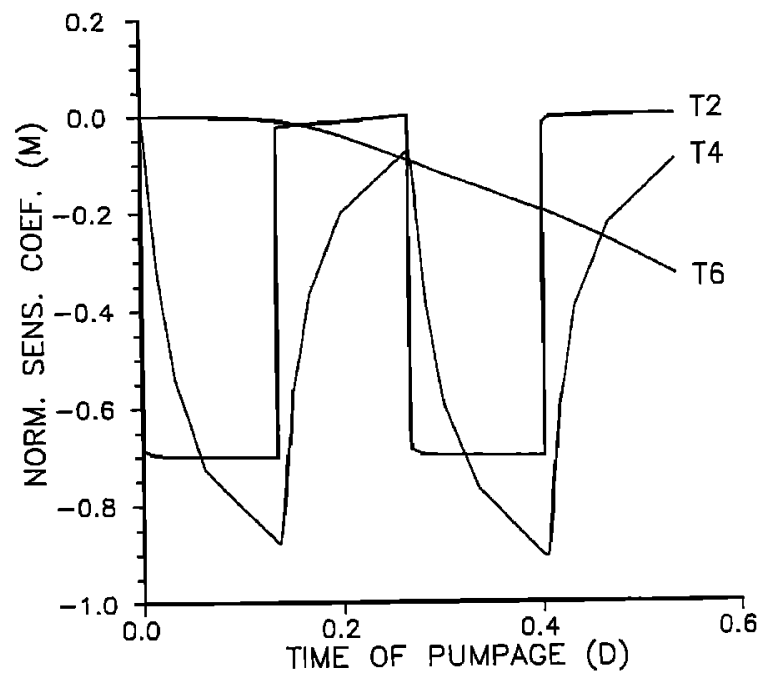

Fig. $10 a$

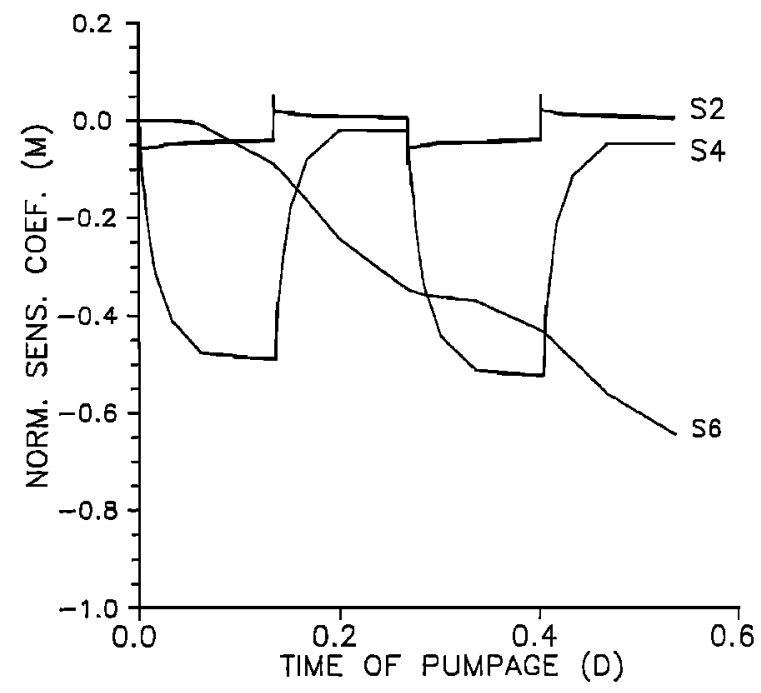

Fig. $10 b$

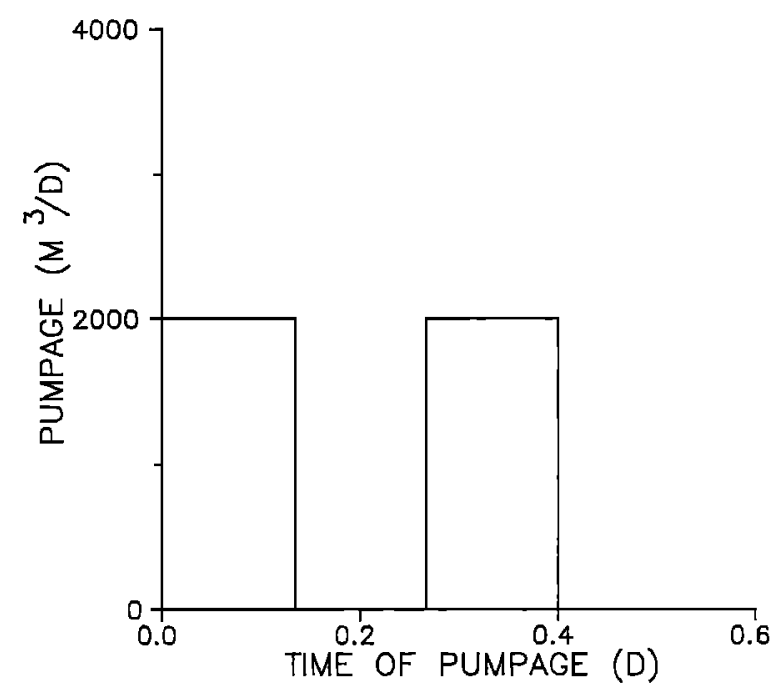

Fig. $10 c$

Fig. 10. (a) Plot of sensitivity versus time for transmissivities in zones 2,4 , and 6 , drawdown measured in zone 1 (sensitivities normalized by multiplication by respective model parameters). (b) Plot of sensitivity versus time for storativities in zones 2,4 , and 6 , drawdown measured in zone 1. (c) Pumpage versus time plot for the case of alternately cutting the pump on and off every 3.2 hours.
TABLE 3. Examples of the Correlation Between Sensitivity Coefficients as a Function of the Pattern of Pumpage

\begin{tabular}{cccr}
\hline Parameter & A & \multicolumn{1}{c}{ B } & C \\
\hline T2-T4 & 0.674 & 0.994 & 0.524 \\
T2-T6 & 0.343 & 0.914 & 0.555 \\
S2-S4 & 0.407 & 0.976 & -0.133 \\
S2-S6 & 0.204 & 0.923 & 0.255 \\
T2-S2 & 0.878 & 0.991 & 0.643 \\
T4-S4 & 0.987 & 1.00 & 0.988 \\
T6-S6 & 0.977 & 0.993 & 0.981 \\
\hline
\end{tabular}

Flow properties as in case 2 of Table 1; observation point is the pumping well. Total period of pumping is 1.07 days. Pumping schedules as follows: A, constant-rate pumpage conditions (1000 $\left.\mathrm{m}^{3} / \mathrm{d}\right) ;$ B, pumpage increased $2000 \mathrm{~m}^{3} / \mathrm{d}$ every 3.2 hours $(2000,4000$, $6000, \cdots) ; C$, pumpage rate varied every 3.2 hours, alternating between $2000 \mathrm{~m}^{3} / \mathrm{d}$ and zero $(2000,0,2000,0, \cdots)$. At each rate change, the size of the interval between measurements is reset to the value of the initial time step $\left(1.0 \times 10^{-6}\right.$ day). Time step acceleration factor equals 2.0 .

addition, such a pattern of pumpage dramatically increases the correlation between sensitivity coefficients for different zonal properties, thus diminishing the effect of the increased sensitivities. Because of the role of increased correlation, the decreases in the estimated standard error of zonal parameters achieved using the pattern of pumpage of Figure $9 c$ can be duplicated by alternately operating the pump at different rates about a mean pumpage. Figure 10 depicts a scheme in which the pump is alternately operating at the initial rate of Figure $9 c$ and shut off. Though the sensitivities are not as large as in Figure 9, a similar decrease in the estimated standard error is obtained because increases in correlation are much smaller for this pattern of pumpage. Table 3 displays examples of the sensitivity correlation relationships (see (A5)) observed with different patterns of pumpage. Comparison of column $B$ with columns $A$ and $C$ demonstrates that continual increases in pumpage significantly increase the degree of correlation between sensitivity coefficients for different zonal properties. A comparison of estimated standard errors for the different pumpage schemes, given in Table 4, shows that the gains that can be realized by continually increasing pumpage or by alternately cutting the pump on and off are approximately the same for the cases considered. In other words, the effective sensitivity gains are nearly equal. Since sensitivity coefficients are proportional to pumpage (see (5) and (6)), these results

TABLE 4. Normalized Estimated Standard Error (Percent) as a Function of the Pattern of Pumpage

\begin{tabular}{cccc}
\hline Parameter & A & B & C \\
\hline T1 & 2.85 & 0.505 & 0.507 \\
T2 & 11.4 & 1.98 & 2.05 \\
T3 & 102. & 18.1 & 18.0 \\
T4 & 51.7 & 8.81 & 8.79 \\
T5 & 23.0 & 4.62 & 3.82 \\
T6 & 17.1 & 3.05 & 2.53 \\
S1 & 11.5 & 2.04 & 2.05 \\
S2 & 342. & 60.5 & 61.1 \\
S3 & 103. & 18.4 & 18.1 \\
S4 & 82.6 & 14.3 & 14.4 \\
S5 & 39.6 & 7.38 & 5.97 \\
S6 & 29.2 & 5.25 & 4.28 \\
\hline
\end{tabular}

Flow properties as in case 2 of Table 1. Pumpage as in Table 3. 


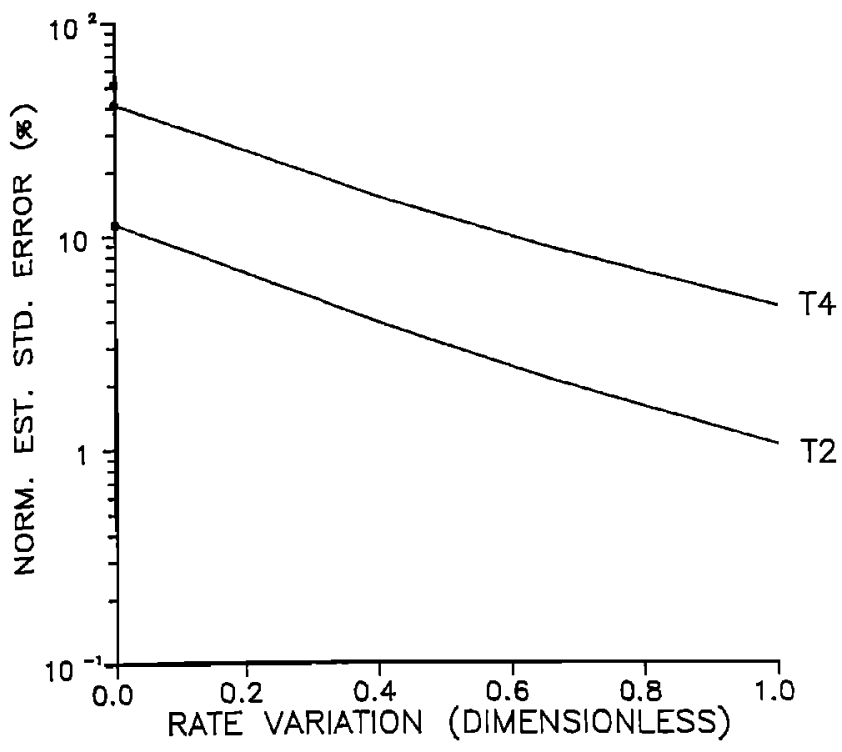

Fig. $11 a$

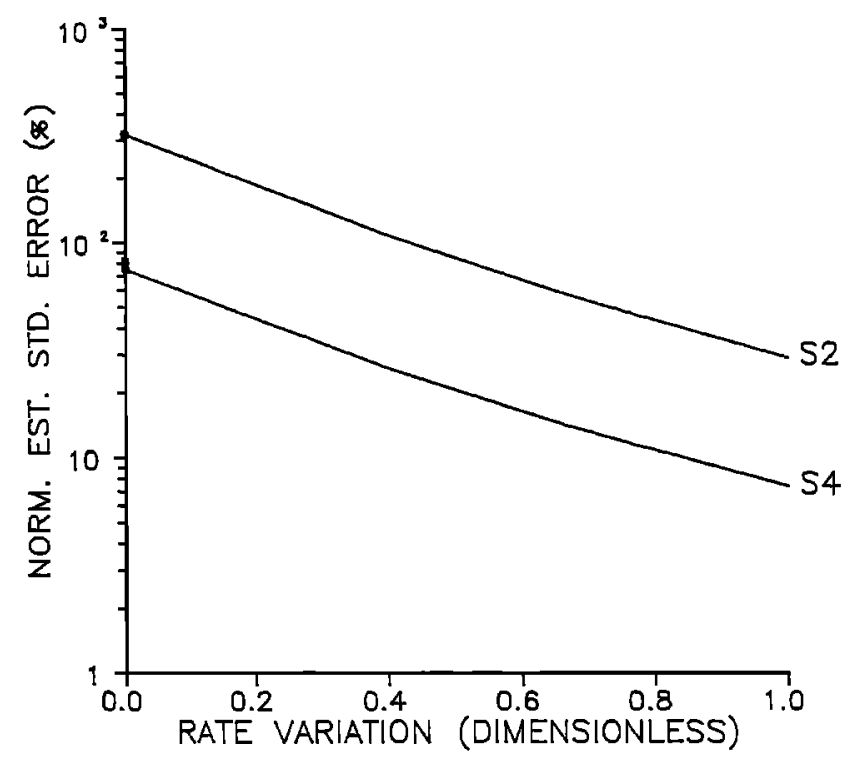

Fig. $11 b$

Fig. 11. Plots of estimated standard error versus magnitude of rate variations for drawdown measured in zone 1 (squares and circles plot on top of each other for zone 2 curves). (a) Results for the transmissivities of zones 2 and 4. (b) Results for the storativities of zones 2 and 4 .

indicate that the effect of the much larger sensitivity coefficients of schedule B is significantly lessened by the large increases in correlation that are produced by that pumping schedule. Note that the results are in keeping with the discussion of Appendix A, which shows how increases in the correlation between sensitivity coefficients will diminish the effect of sensitivity increases.

The capability of a variable-rate pumpage strategy for increasing the effective sensitivity of drawdown to flow properties, by constraining increases in sensitivity correlation, is clear. Variable-rate pumpage in radially symmetric nonuniform aquifers can thus aid in alleviating the non- niqueness problems that arise in pumping test analyses due to insensitivity, measurement error, and correlation. In order to employ the approach effectively, the importance of the magnitude and frequency of the rate changes, and the total duration of the pumping test must be considered in more detail.

\section{Effect of the Magnitude of Pumping Rate Changes}

The plots of Figure 11 display the dependence of the estimated standard error of the zonal parameters on the magnitude of the rate changes for drawdown measured near the pumping well (i.e., within zone 1). In this case, a two-rate pumpage schedule similar to that of Figure $10 \mathrm{c}$ is employed, with the total volume of pumpage in all cases being equivalent to that produced by constant pumpage at $1000 \mathrm{~m}^{3} / \mathrm{d}$ for the duration of the test. Equality in the total volume of pumpage is maintained in this analysis owing to the dependence of sensitivity coefficients on pumpage. The period between rate changes is kept constant at $48 \mathrm{~min}$, with the total duration of the test equal to 25.6 hours. Drawdown measurements are assumed to be taken every time step. In the period between rate changes, a time step acceleration factor of 2.0 is employed. At each rate change, the size of the interval between measurements is reset to the value of the initial time step. The abscissa of Figure 11 is a dimensionless term consisting of the ratio of the difference between the two pumpage rates over the initial rate $((Q 1-Q 2) / Q 1)$. Pumpage at a constant rate thus equals zero, while alternately turning the pump on and off (2000 $\mathrm{m}^{3} / \mathrm{d}$ to 0 in this case) equals 1 . In both plots of Figure 11, the estimated standard error decreases up to an order of magnitude with increases in the size of the rate change. This decrease is a reflection of the interplay between sensitivity and correlation. Note that in both graphs, two values are plotted for each curve at a dimensionless pumpage of zero. The square denotes the constant-pumpage case, with a time step acceleration factor of 2.0 being used since the initial time step. The circled point is for the constant-pumpage case in which the same timestepping scheme is used as in the variable-rate case. The difference between these two points reflects the gains that can be realized by simply taking measurements using a different temporal strategy while pumping at a constant rate. Since the cumulative sensitivity coefficient (the square root of a diagonal element of the sensitivity summation matrix of (A3)) of a circled point is much larger because of the greater number of measurements ( 512 versus 21 ), the small decrease in estimated standard error indicates that the larger correlation accompanying the denser measurement scheme is preventing the sensitivity increases from being fully utilized. The denser measurement scheme increases correlation in the constant-rate case because a large number of measurements are being taken over a small time interval in which sensitivity relationships are changing very little with respect to one another. The larger difference between the two measurement schemes seen within zone 4 parameters is a reflection of the cone front still being within zone 4 when the time step is reset to its initial value, as is explained later.

Figure 12 depicts conditions for drawdown at an observation well in zone 2 at a radial distance of $5.05 \mathrm{~m}$ from the pumping well. The relationships depicted in both plots of Figure 12 are quite similar to those of Figure 11. Distinctly different relationships are found when drawdown at an 


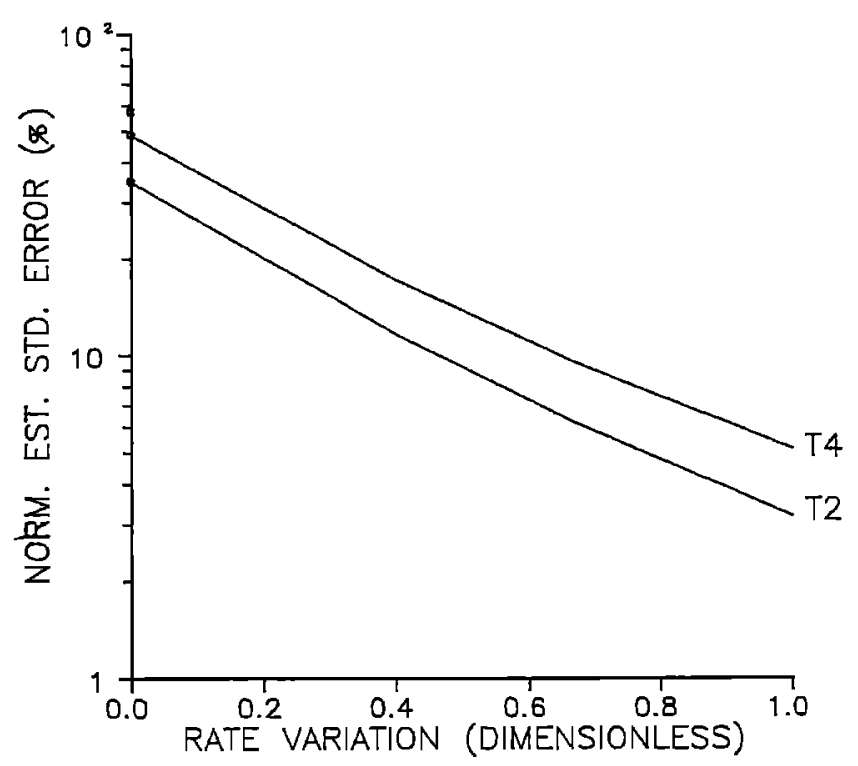

Fig. $12 a$

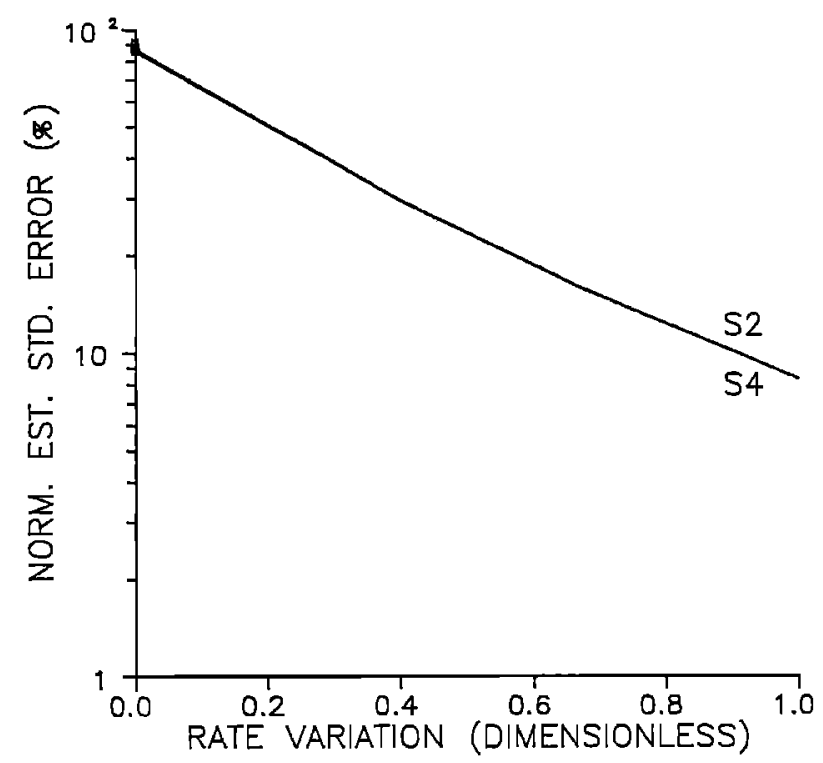

Fig. $12 b$

Fig. 12. Plots of estimated standard error versus magnitude of rate variations for drawdown measured in zone 2 (squares and circles plot on top of each other for zone 2 curves). (a) Results for the transmissivities of zones 2 and 4. (b) Results for the storativities of zones 2 and 4.

observation well in zone 4 (88.4 $\mathrm{m}$ ) is considered (Figure 13). The uncertainty associated with the property estimates of zones 2 and 4 is much greater than that in the earlier figures. The large standard error for parameters in zone 2 is a result of the relative insensitivity of drawdown to properties between the observation well and the pumping well in a radial flow field [see Butler, 1988]. The large error seen for zone 4 estimates is primarily an effect of correlation with zones 1-3. Drawdown at the three observation wells of Figure 11, 12, and 13 does not differ significantly in its sensitivity to properties of zones $4-6$. The estimated standard errors of the parameters in these outer zones, however, depend heavily

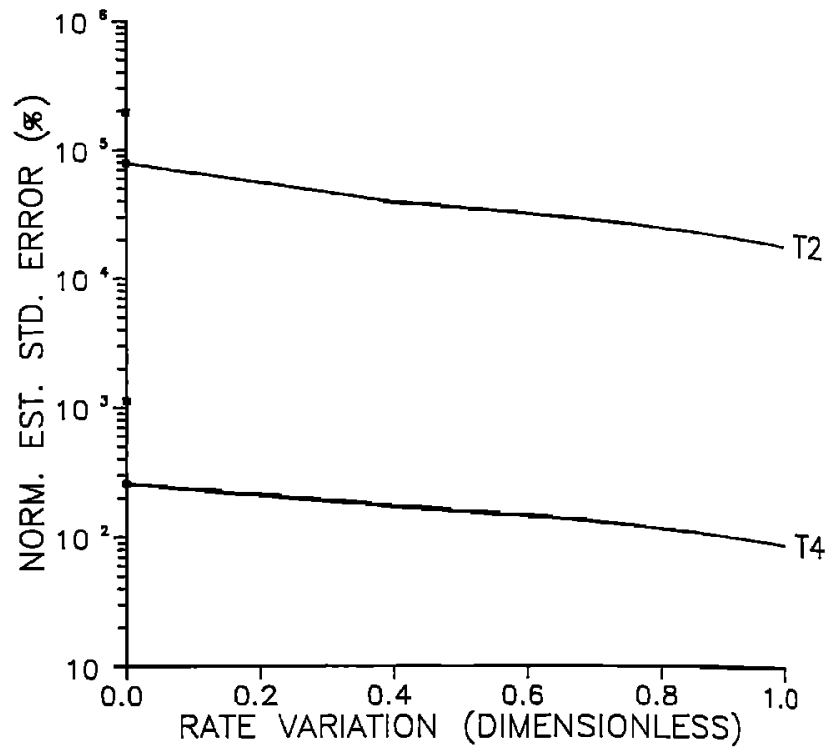

Fig. $13 a$

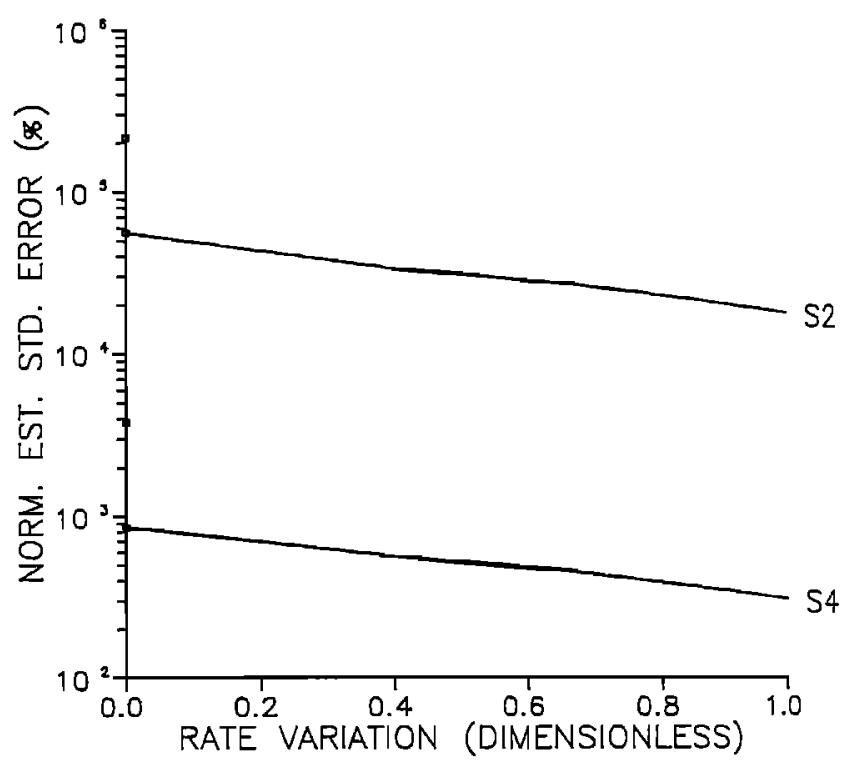

Fig. $13 b$

Fig. 13. Plots of estimated standard error versus magnitude of rate variations for drawdown measured in zone 4. (a) Results for the transmissivities of zones 2 and 4. (b) Results for the storativities of zones 2 and 4 .

on observation well location because of the impact of correlation.

Note that the importance of an observation well is primarily a function of its position; the material of the zone within which the well is sited plays a limited role except in the determination of the properties of that zone. Position here refers both to the position of the observation well with respect to the pumping well as well as to its position within an individual zone. Considerable parameter uncertainty may be introduced as a function of the placement of the observation well within a zone, increasing directly with the outward distance from the inner zonal boundary to the observation well. Thus drawdown measurements at obser- 


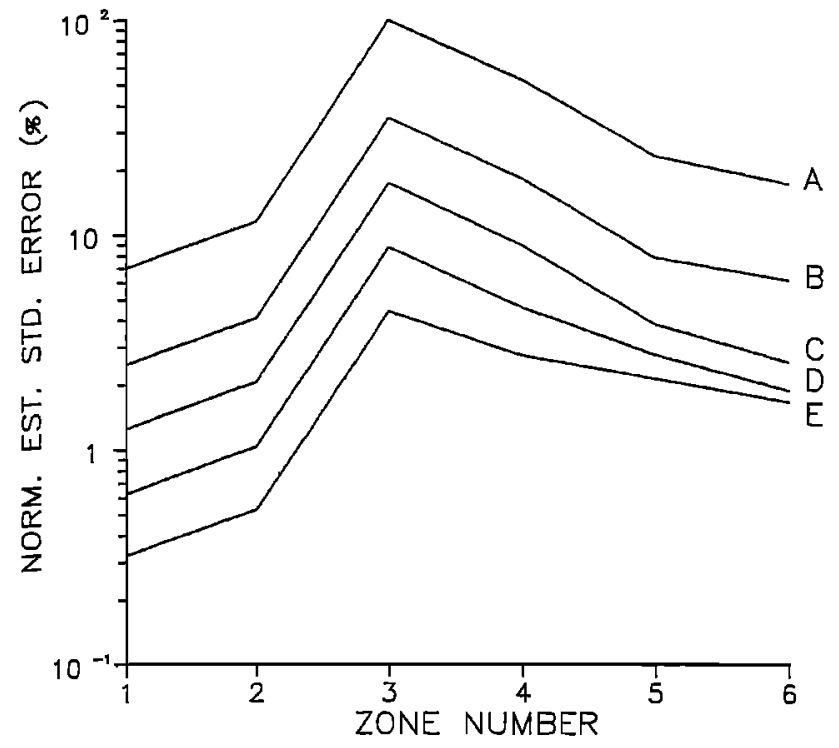

Fig. $14 a$

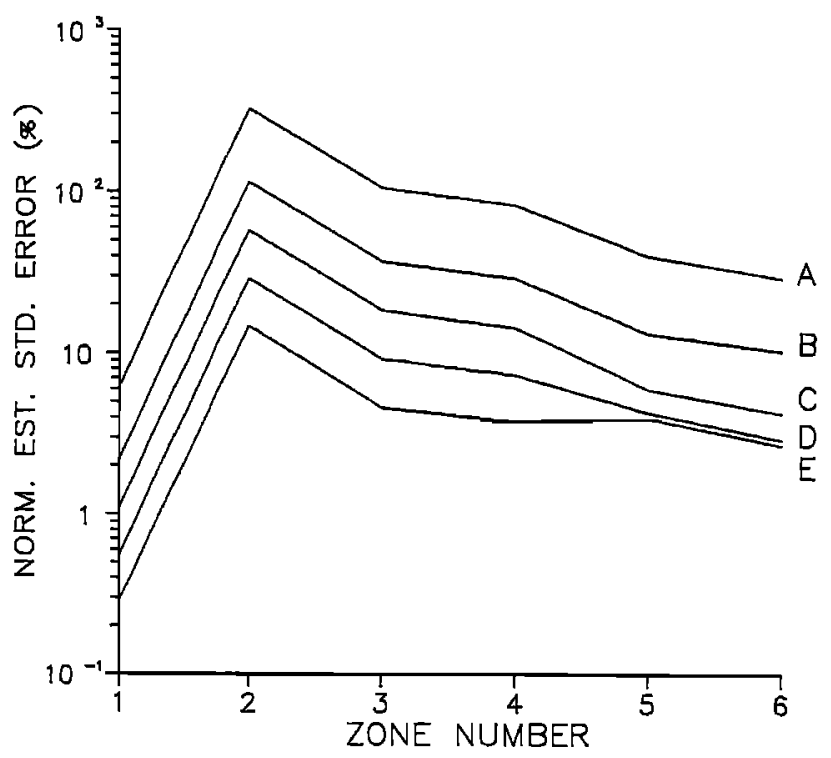

Fig. $14 b$

Fig. 14. Plots of estimated standard error versus zone for five patterns of pumpage $\left(A\right.$, constant pumpage $\left(1000 \mathrm{~m}^{3} / \mathrm{d}\right) ; \mathrm{B}$, rate changed every 12.8 hours $\left(2000 \mathrm{~m}^{3} / \mathrm{d}-0\right)$; C, rate changed every 3.2 hours; D, rate changed every $48 \mathrm{~min}$; E, rate changed every $12 \mathrm{~min}$ ) employing configuration of case 2. Total time of test is 25.6 hours, with drawdown being measured in zone 1. (a) Results for zonal transmissivities. (b) Results for zonal storativities.

vation wells closer to the pumping well are often more useful in estimating the parameters of a distant zone than drawdown measurements at an observation well in the zone itself.

Regardless of the specific conditions under consideration, the basic relationship that emerges from the above analysis is that increases in the magnitude of rate variations decrease the estimated standard error of zonal parameters. Clearly, a pattern of systematic variations of pumpage about a mean value constrains correlation increases to a sufficient extent that the significantly increased cumulative sensitivity coefficients can be more effectively utilized. Note that only dimensionless rate variations less than or equal to 1 were considered here. Under certain conditions, dimensionless variations greater than 1 , in which water is pumped into the aquifer during one of the intervals, could result in still further reductions in parameter uncertainty.

\section{Effect of the Frequency of Pumping Rate Changes}

The results of the previous section pertained to the case of varying pumpage every $48 \mathrm{~min}$. Figure 14 illustrates the dependence of the estimated standard error on the period between rate changes for drawdown at an observation well in zone 1. The total duration of pumpage is again 25.6 hours. Note that the reductions in estimated standard error achieved by decreasing the interval between rate changes are a function of both the increased frequency of rate changes and the increased density of measurements. For zones 1-4 of Figure 14, an increased measurement density in the absence of rate changes improves estimated standard errors only by a small amount because increases in correlation prevent the larger cumulative sensitivity from having an effect. For zones 5 and 6, however, the gains displayed in Figure 14 are largely due to increased measurement density. As is explained shortly, the gains due to an increased density of measurements are a function of the position of the front of the cone of depression when the time step is reset to its initial value. When very high frequencies of rate changes (e.g., 12 and $48 \mathrm{~min}$, curves $\mathrm{E}$ and $\mathrm{D}$, respectively) are employed, the gains due solely to increased measurement density are greater for zone 5 and 6 parameters than those achieved by the pattern of rate variations. This is thought to be primarily a function of the decreasing radius of influence with greater frequencies of rate changes. Note that the points denoting the estimated standard errors for a given schedule of pumpage have been connected in Figure 14 for illustrative purposes. The connecting lines should not be considered to have a physical significance.

The choice of the frequency of pumping rate changes is a function of the portion of the aquifer of interest and the desired detail of the description. Note that there is clearly a practical upper limit to the frequency of rate changes. This practical limit is a function of the time it takes for a pump to reach the desired rate after being turned on, well bore storage, and the amount of flow back down the well after the pump is cut off. Note also that there may be an increase in correlation between sensitivity coefficients for the transmissivity and storativity of the same zone as the frequency of rate changes is increased. This can be seen by considering the differences between the sensitivities to zone 4 properties displayed in Figure 4 and those displayed in Figure 9. The high frequency of rate changes has eliminated the falling limb of the sensitivity to storage curve in Figure $9 b$, resulting in an increase in correlation between the sensitivity coefficients for the transmissivity and storativity of zone 4 . The frequency at which this increased correlation becomes a problem is a function of the position of the zone in the flow field. Thus a test in which the frequency of rate changes is progressively decreased would be an effective means of constraining such correlation increases throughout the aquifer. 


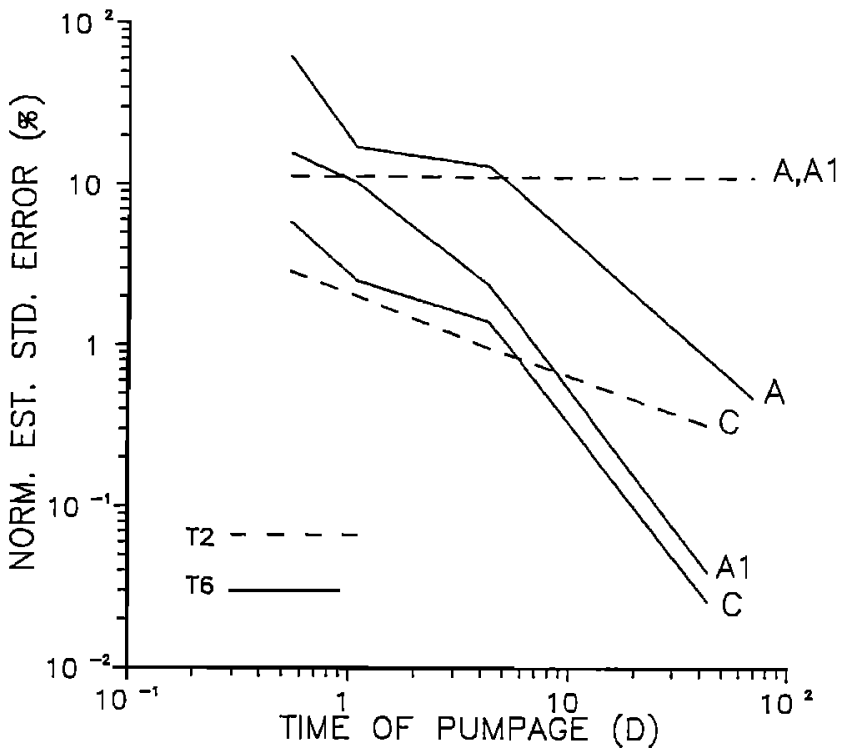

Fig. 15. Plot of estimated standard error of zonal transmissivity versus time of pumpage for three patterns of pumpage ( $A$, constant pumpage $\left(1000 \mathrm{~m}^{3} / \mathrm{d}\right) ; \mathrm{A} 1$, constant pumpage with measurement density of pattern C; C, rate changed every 3.2 hours $\left(2000 \mathrm{~m}^{3} / \mathrm{d}-\right.$ $0)$ ), drawdown measured in zone 1 .

\section{Effect of the Total Duration of Pumpage}

The uncertainty in parameter estimates is also a function of the total duration of the pumping test. Figures 15 and 16 depict the estimated standard error as a function of the total time of pumpage for constant- and variable-rate (rate changes every 3.2 hours) pumpage conditions. The dashed lines in each figure show that the difference between the estimated standard errors for the constant-pumpage and varying-rate cases continually increases for zone 2 properties when drawdown at an observation well in zone 1 is employed. The difference between the constant-pumpage

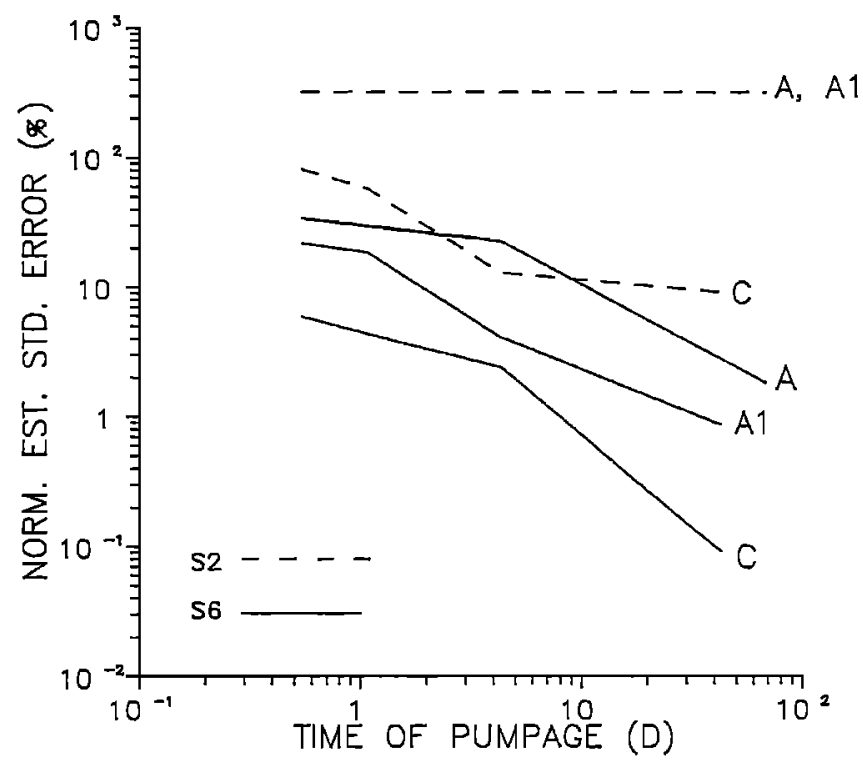

Fig. 16. Plot of estimated standard error of zonal storativity versus time of pumpage for three patterns of pumpage (patterns as in Figure 15), drawdown measured in zone 1. and variable-rate cases, however, is a function of the specific portion of the aquifer under consideration. When an estimate of regional transmissivity is of interest, the difference is much smaller, as shown by the solid lines in Figure 15. In this instance, there is less advantage to varying pumping rates, since the sensitivity of drawdown to regional transmissivity continually increases in both cases. Estimates of regional storativity are improved somewhat more by varying pumping rates, since, as discussed earlier, the sensitivity to regional storativity eventually reaches a constant level in the constant-pumpage case. In general, when the distribution of flow properties is of interest, significant gains can be realized with variable-rate pumping by increasing the total time of pumpage. Note that in both Figures 15 and 16 the curve denoted A1 indicates the estimated standard error for the case of constant pumpage while employing the measurement density of the 3.2-hour variable-rate pumping scheme. In the case of regional transmissivity, the increased measurement density causes curve $A 1$ to converge on curve $C$ (the variable-rate case) at large times, reinforcing the earlier assertion that there is less advantage to varying pumping rates when regional transmissivity is the property of interest. In the case of regional storativity, the convergence of curve Al on the variable-rate case coincides with increases in drawdown sensitivity to regional storativity. Once the sensitivity to storativity curve flattens (e.g., curve $\$ 6$ of Figure 3), curve Al diverges from curve $C$, demonstrating the greater effectiveness of variable-rate pumping for the description of regional storativity.

In Figures 15 and 16 the constant-rate pumpage curve for zone 2 properties is approximately horizontal for all the times plotted. This curve represents both the conventional and the increased measurement density cases. The time at which the curve for the constant-rate case becomes approximately horizontal depends upon the particular zone in question. Essentially, the curve will flatten as soon as the front of the cone of depression has passed out of the zone. As discussed earlier, once the front of the cone of depression has passed out of a given zone, relatively little additional information can be gained concerning the properties of that zone. Appendix B demonstrates that some decrease in the uncertainty of zonal estimates can be expected from measurements taken during the time of constant sensitivity. The experimental simulations of this work have shown, however, that the increased correlation produced by measurements taken during the period of constant sensitivity makes it impossible to realize the gains that would be expected in the absence of such correlation. As implied by the results plotted in Figures 15 and 16, an increased measurement density produces gains over the conventional lower measurement density case as long as the front of the cone of depression has not passed out of the zone of interest. Reference to Figure 10 shows that the cone front has already passed out of zone 2 at 3.2 hours, so an increased measurement density in the constant-pumpage case will not produce substantial gains. In the case of zone 4 , for example, the cone front has not passed out of the zone at 3.2 hours, so an increased density of measurement in the constant-pumping case will produce gains over the conventional lower measurement density case until the cone front has passed out of zone 4 . These gains are illustrated by the differences between the squares and circles in Figures 11-13. The decreases in parameter uncertainty that can be realized by 
simply increasing the measurement density are thus a function of the position of the zone in the radial flow field. Except when zones at a considerable distance from the pumping well are the object of interest, the variable-rate procedure proposed here is superior to constant-rate pumping tests with an increased density of measurements.

\section{SUMMARY AND CONCLUSIONS}

This article has demonstrated that sensitivity analysis can be employed to improve understanding of aquifer behavior during a pumping test and to develop new approaches for estimation of system properties. Based on sensitivity relationships, a variable-rate pumping procedure was proposed here for the characterization of large-scale spatial variations in aquifer properties. This approach enables significant increases in the cumulative sensitivity of drawdown to zonal properties to be realized, while constraining accompanying increases in sensitivity correlation. The effective increase in cumulative sensitivity is thus much larger than that achieved by more conventional approaches. The result is that largescale spatial variations in flow properties can be described in more detail than is normally possible.

Numerical experiments were used here to demonstrate that improvements of over an order of magnitude in the uncertainty of parameter estimates can be readily obtained through the proposed approach. The levels of parameter uncertainty, however, are still considerably higher than those that would be obtained in the absence of correlation between sensitivity coefficients. As demonstrated by the experimental simulations described here, correlation effects can be somewhat controlled by appropriate testing procedures (e.g., the frequency of rate changes can be progressively decreased). In general, however, sensitivity correlation is not as amenable to manipulation as the cumulative sensitivity, as it is often a product of the flow system and the detail of the desired description.

The results of the analyses of this paper also demonstrate the importance of observation well placement for pumping tests. In a radial flow field, an observation well placed near the pumping well allows a record to be established of the properties of the material through which the front of the cone of depression has passed during a pumping test. The detail of the record is a function of the temporal frequency of the drawdown measurements. Drawdown at an observation well placed at a distance from the pumping well is relatively insensitive to material lying between that well and the pumping well, as only the early-time responses are dependent on the properties of the interwell material. Thus observation wells near the pumping well are superior to more distant wells for purposes of characterizing property variability in a radial-flow field.

From the analyses of this work, it is clear that much information concerning subsurface properties can be gained by applying a more rigorous methodology to pumping test design and analysis. Although the recommendations proposed here are based on a one-dimensional analysis, they should be relevant to any flow system in which the primary component of flow is in the radial direction. In order to assess the general applicability of these recommendations to more complex conditions, however, extensions to higher dimensions must be considered. Present research is currently pursuing work in this direction.

\section{APPENDIX A}

In this section, the approach used for the estimation of zonal properties from pumping-induced drawdown is briefly introduced. Following this, the dependence of the estimated standard error of the calculated parameters on the correlation between sensitivity coefficients is examined.

As stated in the body of the paper, the inverse routine used here was an unweighted least squares approach employing sensitivity coefficients. McElwee [1982 $a, 1987]$ describes the approach in considerable detail, so only a summary is given here in order to enable the derivations of this section to be understood.

The approach is an iterative procedure involving the minimization of an error functional in the form of the sum over all observation wells and times of the squared differences between the observed and calculated heads:

$$
E=\sum_{o} \sum_{n}\left[h o_{o}^{n}-h c_{o}^{n}\right]^{2}
$$

where $h o_{o}^{n}$ is the observed head at time $n$ and location $o$ and $h c_{o}^{n}$ is the calculated head at time $n$ and location $o$.

An approximate expression for the calculated head $(h c)$ can be written in terms of sensitivity coefficients as follows:

$$
h c_{o}^{n}=h i_{o}^{n}+\sum_{j} U_{T_{j}}\left(r_{o}, t\right) \Delta T_{j}+\sum_{k} U_{S_{k}}\left(r_{o}, t\right) \Delta S_{k}
$$

where

$$
\begin{aligned}
U_{T_{j}}\left(r_{o}, t\right) & \begin{array}{l}
\text { sensitivity of drawdown at } r_{o} \text { and time } t \text { to } \\
\text { transmissivity in zone } j ;
\end{array} \\
U_{S_{k}}\left(r_{o}, t\right) & \begin{array}{l}
\text { sensitivity of drawdown at } r_{o} \text { and time } t \text { to } \\
\text { storage in zone } k ;
\end{array} \\
h i_{o}^{n} & \begin{array}{l}
\text { head calculated using the initial parameter } \\
\text { vector at time } n \text { and location } o .
\end{array}
\end{aligned}
$$

Following the substitution of (A2) into (A1), the minimization of the functional of (A1) with respect to $T_{j}$ and $S_{k}$ produces a vector of parameter changes, as shown below:

$$
\begin{gathered}
{[A] \Delta \mathbf{P}=\mathbf{R}} \\
\mathbf{\Delta} \mathbf{P}=[A]^{-1} \mathbf{R}
\end{gathered}
$$

where

[A] sensitivity summation matrix;

$M 1$ number of transmissivity zones;

$M$ total number of zones;

$\Delta \mathbf{P}$ vector of parameter changes, $\Delta \mathbf{P}^{+}=\left(\Delta T_{1}, \cdots\right.$, $\left.\Delta T_{M 1}, \Delta S_{M 1+1}, \cdots, \Delta S_{M}\right)$

$\mathbf{k}$ residual vector;

$a_{i j}=\Sigma_{o} \Sigma_{n} U_{T_{i}}\left(r_{o}, n\right) U_{T_{j}}\left(r_{o}, n\right) \quad i, j \leq M 1$

$a_{i j}=\Sigma_{o} \Sigma_{n} U_{T_{1}}\left(r_{o}, n\right) U_{S_{j}}\left(r_{o}, n\right) \quad i \leq M 1 \quad M 1<j \leq M$

$a_{i j}=\Sigma_{o} \Sigma_{n} U_{S_{1}}\left(r_{o}, n\right) U_{T_{j}}\left(r_{o}, n\right) \quad M 1<i \leq M \quad j \leq M 1$

$a_{i j}=\Sigma_{o} \Sigma_{n} U_{S_{1}}\left(r_{o}, n\right) U_{S_{1}}\left(r_{o}, n\right) \quad M 1<i \quad j \leq M$

$r_{k}=\Sigma_{o} \Sigma_{n} U_{T_{k}}\left(r_{o}, n\right)\left(h o_{o}^{n}-h c_{o}^{n}\right) \quad k \leq M 1$

$r_{k}=\Sigma_{o} \Sigma_{n} U_{S_{k}}\left(r_{o}, n\right)\left(h o_{o}^{n}-h c_{o}^{n}\right) \quad M 1<k \leq M$

Since the outlined procedure employs a linear approximation of a nonlinear process, a number of iterations are generally required to obtain convergence to within a predefined criterion.

The elements of the [A] matrix of (A3) and (A4) are summations of products of sensitivity coefficients. The diagonal elements of the matrix are simply summations of the 
squares of the sensitivity coefficients with respect to the same zone (the square roots of the diagonal elements are referred to as cumulative sensitivity coefficients in the text). The $[A]$ matrix can be transformed into a "pseudo" sensitivity-correlation matrix as shown below:

$$
\left[\begin{array}{llll}
1 & C_{12} & \cdots & C_{1 M} \\
C_{21} & 1 & & \vdots \\
\vdots & & \ddots & \vdots \\
C_{M 1} & \cdots & \cdots & 1
\end{array}\right]
$$

where $c_{i j}=a_{i j} /\left(a_{i i} a_{j j}\right)^{1 / 2}$ is a "pseudo" correlation between sensitivity coefficients in zones $i$ and $j,\left|C_{i j}\right|<1$. The elements of this matrix quantify the degree of correlation between sensitivity coefficients characterizing different zonal properties.

Equation (A4) shows that the inverse of the $[A]$ matrix is employed to calculate the vector of parameter changes. The inverse of $[A]$ is also employed to estimate the approximate covariance (A6), as well as the estimated standard error (ESE) of the calculated parameters (A7):

$$
\operatorname{cov}(P)=[A]^{-1} s^{2}=[B]
$$

where

$$
\begin{gathered}
s^{2}=\Sigma_{o} \Sigma_{n}\left[h o_{o}^{n}-h c_{o}^{n}\right]^{2} /\left(\left(N^{*} O\right)-M\right) \\
N \text { total number of time steps; } \\
O \text { total number of observation wells; } \\
{[B] \quad \text { covariance matrix. }}
\end{gathered}
$$

$$
\mathrm{ESE}_{P_{i}}=b_{i i}^{1 / 2}
$$

It is of interest to assess how the correlation between sensitivity coefficients affects the estimated standard error. This relationship is examined here in a rigorous fashion using a two by two matrix and then approximately extended to the $n$ by $n$ case.

Equation (A8) shows a two by two matrix of the summations of the products of the sensitivity coefficients:

$$
\left[\begin{array}{ll}
a_{11} & a_{12} \\
a_{21} & a_{22}
\end{array}\right]
$$

the two diagonal elements. The "pseudo" sensitivitycorrelation matrix corresponding to (A8) is written as

$$
\left[\begin{array}{lc}
1 & C_{12} \\
C_{12} & 1
\end{array}\right]
$$

Since the influence of the correlation between sensitivity coefficients on the estimated standard error is the subject of interest, (A8) can be rewritten in terms of the "pseudo" correlation coefficients of (A9) as

$$
\left[\begin{array}{lr}
a_{11} & C_{12}\left(a_{11} a_{22}\right)^{1 / 2} \\
C_{12}\left(a_{11} a_{22}\right)^{1 / 2} & a_{22}
\end{array}\right]
$$

The inverse of (A10),

$$
\left[\begin{array}{cc}
{\left[a_{11}\left(1-C_{12}^{2}\right)\right]^{-1}} & -\frac{C_{12}}{\left(a_{11} a_{22}\right)^{1 / 2}\left(1-C_{12}^{2}\right)} \\
-\frac{C_{12}}{\left(a_{11} a_{22}\right)^{1 / 2}\left(1-C_{12}^{2}\right)} & {\left[a_{22}\left(1-C_{12}^{2}\right)\right]^{-1}}
\end{array}\right]
$$

clearly shows that increases in correlation between sensitivity coefficients (increases in $\left|C_{12}\right|$ ) lead to an increase in the magnitude of the diagonal elements of $[A]^{-1}$ and thus to an increase in the estimated standard error (A7). Note that the off-diagonal elements of (A11) also indicate that an increase in sensitivity correlation leads to an increase in the absolute magnitude of the correlation between parameters for the two by two case.

Although the matrix-inverse calculations increase in complexity with the order of the matrix, an approximate extension of the above relationships to the $n$ by $n$ case is possible. A three by three matrix is used to illustrate the approximations that are necessary for this extension. Equation (A12) shows the $[A]$ matrix, rewritten in terms of "pseudo" correlation coefficients, for the case of a three by three matrix:

$[A]=\left[\begin{array}{ccc}a_{11} & C_{12}\left(a_{11} a_{22}\right)^{1 / 2} & C_{13}\left(a_{11} a_{33}\right)^{1 / 2} \\ & a_{22} & C_{23}\left(a_{22} a_{33}\right)^{1 / 2} \\ \text { symmetric } & \vdots & a_{33}\end{array}\right]$
The matrix is square and symmetric, with the diagonal elements always being greater than zero and the off-diagonal elements always less than the square root of the product of
It can be shown that the inverse of (A12) can be written in the form of (A13), where $|A|$ is the matrix determinant, which can be expanded as shown in (A14): 
$|A|=a_{11} a_{22} a_{33}\left(1+2 C_{12} C_{13} C_{23}-C_{23}^{2}-C_{13}^{2}-C_{12}^{2}\right)$

If the "pseudo" correlation coefficients are small (i.e., $\left|C_{i j}\right|$ $\ll 1$ ), then all but the lowest powers of the coefficients can be ignored, producing the following approximations of (A14):

$$
\begin{aligned}
|A| & \cong a_{11} a_{22} a_{33}\left[1-C_{23}^{2}-C_{13}^{2}-C_{12}^{2}\right] \\
& \cong a_{11} a_{22} a_{33}\left[1+C_{23}^{2}+C_{13}^{2}+C_{12}^{2}\right]^{-1}
\end{aligned}
$$

Upon substitution of (A15) into (A13), the following form is produced: two by two case. This indicates that the correlation between parameters is a complex nonlinear relationship that cannot be fully expressed by the simple colinearity between two sensitivity coefficients. Equation (A16) shows, however, that if the correlation between sensitivity coefficients is small, then the correlation between parameters will also be small.

For the matrix inverses given in (A11), (A16), and (A17), it is clear that increases in sensitivity may not necessarily produce decreases in the estimated standard error. If the correlation between sensitivity coefficients increases proportionally, the estimated standard error may change very little. This relationship thus helps explain the results discussed in the body of the paper. It is interesting to consider the behavior when all elements of the $[A]$ matrix increase by the same factor. Using principles from elementary linear alge-

$$
[A]^{-1} \cong\left[\begin{array}{lll}
\frac{1}{a_{11}}\left(1+C_{13}^{2}+C_{12}^{2}\right) & \frac{-C_{12}+C_{13} C_{23}}{\left(a_{11} a_{22}\right)^{1 / 2}} & \frac{-C_{13}+C_{12} C_{23}}{\left(a_{11} a_{33}\right)^{1 / 2}} \\
\frac{1}{a_{22}}\left(1+C_{23}^{2}+C_{12}^{2}\right) & \frac{-C_{23}+C_{12} C_{13}}{\left(a_{22} a_{33}\right)^{1 / 2}} \\
\text { symmetric } & & \frac{1}{a_{33}}\left(1+C_{23}^{2}+C_{13}^{2}\right)
\end{array}\right]
$$

Equation (A16) demonstrates that increases in sensitivity correlation $\left(C_{i j}\right)$ lead to increases in the estimated standard enror. Note that the relationships are correct for small $C_{i j}$ to the second order. Numerical experiments done in conjunction with this work demonstrated that the general relationship between increases in sensitivity correlation and increases in estimated standard error appears to hold in systems with $C_{i j}$ of all sizes. Using the same approximations as incorporated in (A16), the $n$ by $n$ equivalent to (A16) can be written as bra, it can be shown that, for a square matrix, the inverse of the product of a matrix and a scalar is simply the reciprocal of the scalar times the inverse of the original matrix. Thus, if the elements of the $[A]$ matrix increase in a proportional fashion, the estimated standard error can decrease significantly. This relationship helps explain the usefulness of the variable-rate procedure proposed here, which allows increases in the elements of the sensitivity-summation matrix $[A]$ while constraining the increases in correlaton.

$$
[A]^{-1} \cong\left[\frac{1}{a_{11}}\left(1+\sum_{j=2}^{M} C_{1 j}^{2}\right)\right.
$$

where only the diagonal elements are given for the sake of clarity. For both the three by three and the $n$ by $n$ cases, the off-diagonal elements are not as easily interpreted as in the

\section{APPENDIX B}

This section examines the change that occurs in the estimated standard error of the transmissivity of a particular 
zone after the cone front leaves that zone. As shown by Figures 2 and $4 a$, the sensitivity coefficient with respect to transmissivity is essentially constant after the cone front moves out of a zone. For a zone near the pumping well, the majority of measurements in a conventional pumping test are taken during the period of constant sensitivity. It is of interest to examine the behavior of the estimated standard error as a function of sampling during this period. If it can be assumed that there is no correlation between sensitivities for different zonal properties and there is only one observation well, then the estimated standard error (ESE) is simply the square root of the product of the reciprocal of the summation of the squared sensitivities times the sample variance as shown below:

$$
\operatorname{ESE}_{T_{i}}=\left[\frac{1}{a_{i i}} \frac{\sum\left[h o^{n}-h c^{n}\right]^{2}}{(N-M)}\right]^{1 / 2}
$$

where

$$
a_{l i}=\sum_{n} U_{T_{i}}^{2}\left(r_{o}, n\right)
$$

Each summation term of (B1) can be rewritten as two components that separately sum the contributions during the periods before and after the cone front moves out of zone $i$ :

$$
\operatorname{ESE}_{T_{i}}=\left[\frac{(N-M)^{-1}\left[C+\left(N-n_{1}\right) D\right]}{E+\left(N-n_{1}\right) F}\right]^{-1 / 2}
$$

where $n_{1}$ is the time at which the cone front can be assumed to have passed out of zone $i, U_{T}$ and $\left[h o^{n}-h c^{n}\right]$ are assumed constant after this time, and

$$
\begin{gathered}
C=\sum_{n=1}^{n_{1}}\left[h o^{n}-h c^{n}\right]^{2} \\
D \cong\left[h o^{n}-h c^{n}\right]^{2} \cong \mathrm{const} \quad n>n_{1} \\
\left(N-n_{1}\right) D \cong \sum_{n=n_{1}+1}^{N}\left[h o^{n}-h c^{n}\right]^{2} \\
E=\sum_{n=1}^{n_{1}} U_{T_{1}}^{2}\left(r_{o}, n\right) \\
F \cong U_{T_{1}}^{2}\left(r_{o}, n\right) \cong \operatorname{const}^{N} n>n_{1} \\
\left(N-n_{1}\right) F \cong \sum_{n=n_{1}+1}^{N} U_{T_{i}}^{2}\left(r_{o}, n\right)
\end{gathered}
$$

If the vast majority of measurements are collected during the period of constant sensitivity for zone $i$, it can be assumed that $\left(N-n_{1}\right) F \gg E$ and $\left(N-n_{1}\right) D \gg C$. This allows (B2) to be approximated as

$$
\begin{aligned}
\operatorname{ESE}_{T_{1}} & \cong\left[\frac{D}{(N-M) F}\right]^{1 / 2} \\
& \cong \frac{1}{N^{1 / 2}} \text { (const) }
\end{aligned}
$$

Equation (B3) indicates that the maximum rate at which the estimated standard error can decrease is approximately proportional to the inverse square root of the number of measurements. Thus a very large number of measurements must be taken to get a significant decrease in the estimated standard error. Equation (B3), however, was developed in the absence of correlation between sensitivity coefficients, a condition not generally met in radial flow systems. Equa. tions such as (A17) indicate that correlation should have a significant impact on the rate of decrease of the estimated standard error. This is supported by the experimental simulations of this study, which demonstrate that when sensitivity correlation is large, the rate of decrease in the estimated standard error is much smaller than the maximum value given in (B3). Increased measurement density during the period of constant sensitivity thus usually leads to very small gains in the estimated standard error. Note that the sensitivity of drawdown to the transmissivity of a zone far from the pumping well may not reach a constant value during the period of pumping (e.g., curves T4 and T6 of Figure 4a). For such a zone an increased measurement density produces a significant decrease in the estimated standard error, since the diagonal elements of the sensitivity-summation matrix are increasing in size faster than the correlation. The differences between the squares and circles in the plots for the fourth zone in Figures 11-13 are examples of this behavior.

\section{References}

Barker, J. A., and R. Herbert, Pumping tests in patchy aquifers, Ground Water, 20(2), 150-155, 1982.

Beck, J. V., and K. J. Arnold, Parameter Estimation in Engineering and Science, John Wiley, New York, 1977.

Butler, J. J., Jr., Pumping tests in nonuniform aquifers: A deterministic/stochastic analysis, Ph.D. thesis, 220 pp., Stanford Univ., Stanford, Calif., 1986.

Butler, J. J., Jr., Pumping tests in nonuniform aquifers: The radially symmetric case, J. Hydrol., 101(1/4), 15-30, 1988.

de Marsily, G., Spatial variability: Ovid's Metamorphoses revisited, paper presented at IAHS Workshop on Spatial Variability and Representativeness of Hydrogeological Parameters, IUGG Gen. Assem., Vancouver, B. C., Canada, 1987.

McElwee, C. D., A one-dimensional study of the groundwater inverse problem using sensitivity analysis, Open File Rep. 82-12, Kansas Geol. Surv., Lawrence, $1982 a$.

McElwee, C. D., Sensitivity analysis and the ground-water inverse problem, Ground Water, 20(6), 723-735, $1982 b$.

McElwee, C. D., Sensitivity analysis of groundwater models, Advances in Transport Phenomena in Porous Media, edited by J. Bear and M. Y. Corapcioglu, NATO Adv. Study Inst. Ser., Ser. E, 128, 751-817, 1987.

McElwee, C. D., and M. A. Yukler, Sensitivity of groundwater models with respect to variations in transmissivity and storage, Water Resour. Res., 14(3), 451-459, 1978.

Rushton, K. R., and Y.-K. Chan, Numerical pumping test analysis in unconfined aquifers, J. Irrig. Drain. Div. Am. Soc. Civ. Eng., 103(IR1), 1-12, 1977.

Streltsova, T. D., Well Testing in Heterogeneous Formations, John Wiley, New York, 1988.

Toth, J., Hydrogeology and yield evaluation of a municipal well field, Alberta, Canada, Hydrol. Sci. J., 18(2), 165-189, 1973.

Yeh, W. W.-G., and N.-Z. Sun, An extended identifiability in aquifer parameter identification and optimal pumping test design, Water Resour. Res., 20(12), 1837-1847, 1984.

J. J. Butler, Jr., and C. D. McElwee, Kansas Geological Survey, University of Kansas, 1930 Constant Avenue, Lawrence, KS 66046.

(Received July 27, 1988; revised July 28, 1989; accepted August 3, 1989.) 